\title{
Maximal and Stabilizing Hermitian Solutions for Discrete-Time Coupled Algebraic Riccati Equations
}

\author{
O. L. V. Costa and R. P. Marques \\ University of São Paulo, Department of Electronic Engineering \\ Av. Prof. Luciano Gualberto - travessa 3, 158 \\ 05508-900 São Paulo SP Brazil \\ phone: +55.11.818-5771 - fax: +55.11.818-5718 \\ email: oswaldo@lac.usp.br; rpm@lac.usp.br
}

\begin{abstract}
Discrete-time coupled algebraic Riccati equations that arise in quadratic optimal control and $H_{\infty}$-control of Markovian jump linear systems are considered. First, the equations that arise from the quadratic optimal control problem are studied. The matrix cost is only assumed to be hermitian. Conditions for existence of the maximal hermitian solution are derived in terms of the concept of mean square stabilizability and a convex set not being empty. A connection with convex optimization is established, leading to a numerical algorithm. A necessary and sufficient condition for existence of a stabilizing solution (in the mean square sense) is derived. Sufficient conditions in terms of the usual observability and detectability tests for linear systems are also obtained. Finally, the coupled algebraic Riccati equations that arise from the $H_{\infty}$-control of discrete-time Markovian jump linear systems are analyzed. An algorithm for deriving a stabilizing solution, if it exists, is obtained. These results generalize and unify several previous ones presented in the literature of discretetime coupled Riccati equations of Markovian jump linear systems.
\end{abstract}

Keywords: maximal hermitian solutions, stabilizing solutions, coupled algebraic Riccati equations, quadratic optimal control, $H_{\infty}$-control.

November 1996; revised in July 1998; January 1999. 


\section{Introduction}

Algebraic Riccati equations arise in control theory as an important tool for solving many optimization control and filtering problems, in particular the linear-quadratic and $H_{\infty}$-control problems, and have been extensively studied in the current literature (see, for instance, $[\mathrm{BLW}],[\mathrm{RV}],[\mathrm{RT}],[\mathrm{SSC}],[\mathrm{SW}],[\mathrm{W} 2])$. One of the most successful methods for analyzing the problem is via geometric methods, in which deflating subspaces (sometimes also called invariant subspaces) of the so-called extended sympletic matrix pencil are considered (see for instance $[B L W])$. Usually one is interested in a stabilizing solution, that is, the solution that gives rise to a state feedback matrix which stabilizes the system in closed loop. In this context the maximal solution, which will coincide with the stabilizing solution whenever it exists, is also of interest. Some papers are devoted to characterize such solutions and we can mention, in particular, [OZ] and $[\mathrm{RV}]$, where a sufficient condition, written in terms of a convex set not being empty and the concept of stabilizability, is derived for the existence of the maximal hermitian solution of a Riccati equation.

Discrete-time coupled algebraic Riccati equations (CARE) that arise in quadratic optimal control and $H_{\infty}$-control of Markovian jump linear systems are considered in this paper. First, we consider the quadratic optimal control problem. Such equations arise when one desires to minimize the following functional

$$
J(x(0), \theta(0), u)=\frac{1}{2} \sum_{k=0}^{\infty} \mathrm{E}\left\{\left[\begin{array}{ll}
x(k)^{*} & u(k)^{*}
\end{array}\right]\left[\begin{array}{cc}
Q_{\theta(k)} & L_{\theta(k)} \\
L_{\theta(k)}^{*} & R_{\theta(k)}
\end{array}\right]\left[\begin{array}{l}
x(k) \\
u(k)
\end{array}\right]\right\}
$$

where

$$
x(k+1)=A_{\theta(k)} x(k)+B_{\theta(k)} u(k)
$$

and $\theta(k)$ denotes a Markov chain taking values in $\{1, \ldots, N\}$ with transition probability matrix $\mathbb{P}=\left[p_{i j}\right]$. In most cases the matrices $\left[\begin{array}{ll}Q_{i} & L_{i} \\ L_{i}^{*} & R_{i}\end{array}\right]$ are positive semi-definite for $i=1, \ldots, N$, but the indefinite case is also of interest for quadratic control (see for instance, [D],[FCS] for the deterministic quadratic discrete-time optimal control problem). Models as above are known in the current literature as discrete time Markovian jump linear systems, and have been extensively studied recently. This follows in part due to the fact that a great number of dynamic systems are vulnerable to abrupt changes in their structures caused by failures, sudden environmental changes, variation of the operating point of a nonlinear plant, etc. Several aspects related to applications, stability theory, optimal control theory and $H_{\infty}$-control of these systems can be found nowadays in the literature (see, for instance, [C],[CF1],[CV1],[CF2],[CV2],[JFL],[M1],[M2],[M3],[RG]).

The linear quadratic optimal control problem of Markovian jump linear systems with $L_{i}=0$, $Q_{i} \geq 0, R_{i}>0$, and the associated CARE, have been studied in [C],[CF2],[JFL],[M2],[RG]. Conditions for existence of the maximal solution and stabilizing solution (in the mean square sense, as will be defined in Definition 1 below) were presented in [C],[CF2] in terms of the concept of mean square stabilizability and mean square detectability. Mean square stability can be replaced by some conditions on the unobservable modes of the system (see [C],[M2]) or stochastic observability [JFL]. Characterization of the maximal solution in terms of a LMI optimization problem has been presented in [RG]. Continuous time results related to the problem posed here 
can be found in [FCS] and [W3], where conditions for existence of maximal and stabilizing solution of a linearly perturbed Riccati equation are presented. In this paper we unify and generalize all previous results related to the quadratic CARE. The matrix cost is only assumed to be Hermitian. When restricted to the case in which $L_{i}=0, Q_{i} \geq 0, R_{i}>0$, the conditions derived here generalize the previous ones. The CARE that arise in the $H_{\infty}$-control of Markovian jump linear systems (cf. [CV1]) is also studied, and an algorithm for deriving a stabilizing solution, whenever it exists, is presented.

This paper is organized in the following way. Section 2 presents some preliminary results and the notation that will be adopted throughout the paper. Section 3 presents sufficient conditions for the existence of the maximal solution for the CARE associated to the quadratic optimal control problem (Theorem 1), as well as a representation of this solution in terms of the solution of a convex optimization problem (Theorem 2). Section 4 deals with necessary and sufficient conditions for the existence of a stabilizing solution (Theorems 3 and 4). Section 5 presents a recursive procedure for obtaining a stabilizing solution of the CARE associated to the $H_{\infty}$-control problem, whenever it exits (Theorem 6). The paper is concluded in Section 6 with some final comments. The proofs of some auxiliary results are presented in the Appendix.

\section{Notation and Preliminary Results}

For $\mathbb{X}$ and $\mathbb{Y}$ complex Banach spaces we set $\mathbb{B}(\mathbb{X}, \mathbb{Y})$ the Banach space of all bounded linear operators of $\mathbb{X}$ into $\mathbb{Y}$, with the uniform induced norm represented by $\|$.$\| . For simplicity we shall$ set $\mathbb{B}(\mathbb{X}):=\mathbb{B}(\mathbb{X}, \mathbb{X})$. The spectral radius of an operator $\mathcal{T} \in \mathbb{B}(\mathbb{X})$ will be denoted by $r_{\sigma}(\mathcal{T})$. If $\mathbb{X}$ is a Hilbert space then the inner product will be denoted by $\langle. ;$.$\rangle , and for \mathcal{T} \in \mathbb{B}(\mathbb{X}), \mathcal{T}^{*}$ will denote the adjoint operator of $\mathcal{T}$. As usual, $\mathcal{T} \geq 0(\mathcal{T}>0$ respectively) will denote that the operator $\mathcal{T} \in \mathbb{B}(\mathbb{X})$ will be positive semi-definite (positive definite). In particular we shall denote by $\mathbb{C}^{n}$ the $n$ dimensional complex Euclidean spaces and by $\mathbb{B}\left(\mathbb{C}^{n}, \mathbb{C}^{m}\right)$ the normed bounded linear space of all $m \times n$ complex matrices, with $\mathbb{B}\left(\mathbb{C}^{n}\right):=\mathbb{B}\left(\mathbb{C}^{n}, \mathbb{C}^{n}\right)$.

Set $\mathbb{H}^{n, m}$ the linear space made up of all $N$-sequences of complex matrices $V=\left(V_{1}, \ldots, V_{N}\right)$ with $V_{i} \in \mathbb{B}\left(\mathbb{C}^{n}, \mathbb{C}^{m}\right), \quad i=1, \ldots, N \quad$ and, for simplicity, set $\mathbb{H}^{n}:=\mathbb{H}^{n, n}$. For $V=\left(V_{1}, \ldots, V_{N}\right) \in \mathbb{H}^{n, m}$, we consider the following norms in $\mathbb{H}^{n, m}$

$$
\begin{gathered}
\|V\|_{1}:=\sum_{i=1}^{N}\left\|V_{i}\right\| \\
\|V\|_{2}:=\left(\sum_{i=1}^{N} \operatorname{tr}\left(V_{i}^{*} V_{i}\right)\right)^{\frac{1}{2}}
\end{gathered}
$$

It is easy to verify that $\mathbb{H}^{n, m}$ equipped with any of the above norms is a Banach space and, in fact, $\left(\|\cdot\|_{2}, \mathbb{H}^{n, m}\right)$ is a Hilbert space, with the inner product given, for $V=\left(V_{1}, \ldots, V_{N}\right)$ and $S=\left(S_{1}, \ldots, S_{N}\right)$ in $\mathbb{H}^{n, m}$, by

$$
\langle V ; S\rangle:=\sum_{i=1}^{N} \operatorname{tr}\left(V_{i}^{*} S_{i}\right) .
$$


We shall say that $V=\left(V_{1}, \ldots, V_{N}\right) \in \mathbb{H}^{n}$ is hermitian if $V_{i}=V_{i}^{*}$ for $i=1, \ldots, N$, and denote this set by $\mathbb{H}^{n *}$. We shall write $\mathbb{H}^{n+}:=\left\{V=\left(V_{1}, \ldots, V_{N}\right) \in \mathbb{H}^{n *} ; V_{i} \geq 0, i=\right.$ $1, \ldots, N\}$ and for $V \in \mathbb{H}^{n}, \quad S \in \mathbb{H}^{n}$, we write that $V \geq S$ if $V-S=\left(V_{1}-S_{1}, \ldots, V_{N}-S_{N}\right) \in \mathbb{H}^{n+}$, and that $V>S$ if $V_{i}-S_{i}>0$ for $i=1, \ldots, N$.

For $\Gamma=\left(\Gamma_{1}, \ldots, \Gamma_{N}\right) \in \mathbb{H}^{n} \quad$ and matrix $\mathbb{P}=\left[p_{i j}\right], i, j=1, \ldots, N$, with $p_{i j} \geq 0$ for all $i, j=1, \ldots, N$, we define the following operators $\mathcal{E}()=.\left(\mathcal{E}_{1}(),. \ldots, \mathcal{E}_{N}().\right) \in \mathbb{B}\left(\mathbb{H}^{n}\right)$, $\mathcal{L}()=.\left(\mathcal{L}_{1}(),. \ldots, \mathcal{L}_{N}().\right) \in \mathbb{B}\left(\mathbb{H}^{n}\right)$ and $\mathcal{T}()=.\left(\mathcal{T}_{1}(),. \ldots, \mathcal{T}_{N}().\right) \in \mathbb{B}\left(\mathbb{H}^{n}\right) ;$

$$
\begin{gathered}
\mathcal{E}_{i}(V):=\sum_{j=1}^{N} p_{i j} V_{j} \\
\mathcal{L}_{i}(V):=\Gamma_{i}^{*} \mathcal{E}_{i}(V) \Gamma_{i} \\
\mathcal{T}_{j}(V):=\sum_{i=1}^{N} p_{i j} \Gamma_{i} V_{i} \Gamma_{i}^{*}
\end{gathered}
$$

where $V=\left(V_{1}, \ldots, V_{N}\right) \in \mathbb{H}^{n}$. It is easy to verify that with the inner product given by (2.c) above we have that $\mathcal{T}=\mathcal{L}^{*}$. It is also easy to check that the operators $\mathcal{E}, \mathcal{L}$, and $\mathcal{T}$ map $\mathbb{H}^{n *}$ into $\mathbb{H}^{n *}$ and $\mathbb{H}^{n+}$ into $\mathbb{H}^{n+}$.

For $A=\left(A_{1}, \ldots, A_{N}\right) \in \mathbb{H}^{n} \quad$ and $B=\left(B_{1} \ldots, B_{N}\right) \in \mathbb{H}^{m, n}$ consider model (1) with $u(k)=F_{\theta(k)} x(k)$, where $F=\left(F_{1}, \ldots, F_{N}\right) \in \mathbb{H}^{n, m}$. It has been shown in [CF1] that, for $V_{i}(k)=\mathrm{E}\left(x(k) x(k)^{*} 1_{\{\theta(k)=i\}}\right), V(k)=\left(V_{1}(k), \ldots, V_{N}(k)\right) \in \mathbb{H}^{n+}$, we have that

$$
V(k+1)=\mathcal{T}(V(k)), k=0,1, \ldots
$$

where $\Gamma_{i}=A_{i}+B_{i} F_{i}$ in (3.c). We define next the stability and stabilizability concepts that we shall consider in the following sections.

Definition 1: We say that $F=\left(F_{1}, \ldots, F_{N}\right) \in \mathbb{H}^{n, m}$ stabilizes $(A, B)$ in the mean square sense if, when we make $u(k)=F_{\theta(k)} x(k)$ in system (1), we have that $\mathrm{E}\left(\|x(k)\|^{2}\right) \rightarrow 0$ as $k \rightarrow \infty$ for any initial condition $x(0)$ and $\theta(0)$.

Definition 2: We say that $(A, B)$ is mean square stabilizable if for some $F=\left(F_{1}, \ldots, F_{N}\right) \in \mathbb{H}^{n, m}$, we have that $F$ stabilizes $(A, B)$ in the mean square sense.

The following result, proved in [3], shows that $F=\left(F_{1}, \ldots, F_{N}\right)$ stabilizes system (1) in the mean square sense if and only if the expectral radius of the operator (3.c) in closed loop is less than one.

Lemma 1: $F=\left(F_{1}, \ldots, F_{N}\right) \in \mathbb{H}^{n, m}$ stabilizes $(A, B)$ in the mean square sense if and only if $r_{\sigma}(\mathcal{T})<1$, where $\mathcal{T}$ is as in (3.c) with $\Gamma_{i}=A_{i}+B_{i} F_{i}$.

We make the following definition, similar to the detectability concept for deterministic systems. 
Definition 3: Consider $\mathcal{C}=\left(\mathcal{C}_{1}, \ldots, \mathcal{C}_{N}\right) \in \mathbb{H}^{n, r}$. We say that $(\mathcal{C}, A)$ is mean square detectable if for some $H=\left(H_{1}, \ldots, H_{N}\right) \in \mathbb{H}^{r, n}, r_{\sigma}\left(\mathcal{L}<1\right.$, where $\mathcal{L}()=.\left(\mathcal{L}_{1}(),. \ldots, \mathcal{L}_{N}().\right)$ is as in (3.b) above with $\Gamma_{i}=A_{i}+H_{i} \mathcal{C}_{i}, i=1, \ldots, N$.

The next lemma, proved in the Appendix, will be crucial for the development of the paper. Consider also the operator $\overline{\mathcal{L}}()=.\left(\overline{\mathcal{L}}_{1}(),. \ldots, \overline{\mathcal{L}}_{N}().\right) \in \mathbb{B}\left(\mathbb{H}^{n}\right)$ defined as

$$
\overline{\mathcal{L}}_{i}(.):=\Lambda_{i}^{*} \mathcal{E}_{i}(.) \Lambda_{i}, i=1, \ldots, N
$$

where $\Lambda_{i}=A_{i}+B_{i} G_{i}, i=1, \ldots, N$, for some $G=\left(G_{1}, \ldots, G_{N}\right) \in \mathbb{H}^{n, m}$.

Lemma 2: Let $\mathcal{L}$ and $\overline{\mathcal{L}}$ be as defined in (3.b) and (4) above, with $\Gamma_{i}=A_{i}+B_{i} F_{i}$ and $\Lambda_{i}=A_{i}+B_{i} G_{i}, \quad i=1, \ldots, N$. Suppose that $r_{\sigma}(\mathcal{L})<1$ and for some $P=\left(P_{1}, \ldots, P_{N}\right) \geq 0$ and $\delta>0$,

$$
P_{i}-\Lambda_{i}^{*} \mathcal{E}_{i}(P) \Lambda_{i} \geq \delta\left(G_{i}-F_{i}\right)^{*}\left(G_{i}-F_{i}\right), i=1, \ldots, N .
$$

Then $r_{\sigma}(\overline{\mathcal{L}})<1$.

Finally we conclude this section with the next lemma (see proof in the Appendix).

Lemma 3: Consider $S=\left(S_{1}, \ldots, S_{N}\right) \in \mathbb{H}^{n}$. Suppose that $r_{\sigma}(\mathcal{L})<1$, where $\mathcal{L}($.$) is as$ defined in (3) above. Then there exist a unique solution $Y=\left(Y_{1}, \ldots, Y_{N}\right) \in \mathbb{H}^{n}$ which satisfies

$$
Y_{i}-\Gamma_{i}^{*} \mathcal{E}_{i}(Y) \Gamma_{i}=S_{i}, i=1, \ldots, N .
$$

Moreover if $S$ is hermitian ( $\geq 0,>0$ respectively) then $Y$ is hermitian $(\geq 0,>0)$. On the other hand if for some $S>0$ there exists $Y>0$ satisfying equation (6) then $r_{\sigma}(\mathcal{L})<1$.

Remark 1: From Lemma 3 it is clear that if $r_{\sigma}(\mathcal{L})<1$ then from the standard discrete-time Lyapunov equation we have that $r_{\sigma}\left(p_{i i}^{1 / 2} \Gamma_{i}\right)<1$ for each $i=1, \ldots, N$.

\section{Maximal Solution}

Consider $L=\left(L_{1}, \ldots, L_{N}\right) \in \mathbb{H}^{m, n}, Q=\left(Q_{1}, \ldots, Q_{N}\right) \in \mathbb{H}^{n *}$ and $R=\left(R_{1} \ldots, R_{N}\right) \in \mathbb{H}^{m *}$. Therefore, regarding $Q$ and $R$, we only assume that they are hermitian.We will study the following set of coupled algebraic Riccati equations (CARE):

$$
0=-X_{i}+A_{i}^{*} \mathcal{E}_{i}(X) A_{i}+Q_{i}-\left(A_{i}^{*} \mathcal{E}_{i}(X) B_{i}+L_{j}\right)\left(B_{i}^{*} \mathcal{E}_{i}(X) B_{i}+R_{i}\right)^{-1}\left(B_{i}^{*} \mathcal{E}_{i}(X) A_{i}+L_{i}^{*}\right)
$$

and we shall say that $X=\left(X_{1} \ldots, X_{N}\right) \in \mathbb{H}^{n *}$ will be a hermitian solution for the CARE if $B_{i}^{*} \mathcal{E}_{i}(X) B_{i}+R_{i}$ is invertible and $X$ satisfies the equation above.

Set

$$
\mathbb{L}:=\left\{X=\left(X_{1}, \ldots, X_{N}\right) \in \mathbb{H}^{n *} ;\left(B_{i}^{*} \mathcal{E}_{i}(X) B_{i}+R_{i}\right)^{-1} \text { exists for } i=1, \ldots, N\right\} .
$$

We define the following nonlinearoperator $\mathcal{R}()=.\left(\mathcal{R}_{1}(),. \ldots, \mathcal{R}_{N}().\right): \mathbb{L} \rightarrow \mathbb{H}^{n}$. 
Definition 4: For $X=\left(X_{1}, \ldots, X_{N}\right) \in \mathbb{L}$, we define $\mathcal{R}(X)=\left(\mathcal{R}_{1}(X), \ldots, \mathcal{R}_{N}(X)\right)$ as

$$
\mathcal{R}_{i}(X)=-X_{i}+A_{i}^{*} \mathcal{E}_{i}(X) A_{i}+Q_{i}-\left(A_{i}^{*} \mathcal{E}_{i}(X) B_{i}+L_{i}\right)\left(B_{i}^{*} \mathcal{E}_{i}(X) B_{i}+R_{i}\right)^{-1}\left(B_{i}^{*} \mathcal{E}_{i}(X) A_{i}+L_{i}^{*}\right) .
$$

We introduce the following notation

$$
\begin{aligned}
& \mathbb{N}:=\left\{X=\left(X_{1}, \ldots, X_{N}\right) \in \mathbb{L} \text { and } B_{i}^{*} \mathcal{E}_{i}(X) B_{i}+R_{i}>0, i=1, \ldots, N\right\} \\
& \mathbb{M}:=\left\{X=\left(X_{1}, \ldots, X_{N}\right) \in \mathbb{N}, \mathcal{R}(X) \geq 0\right\} \\
& \widehat{\mathbb{M}}:=\left\{X=\left(X_{1}, \ldots, X_{N}\right) \in \mathbb{N}, \mathcal{R}(X)=0\right\} \text {. }
\end{aligned}
$$

As we shall see in the proof of Theorem 2, $\mathbb{M}$ can be written as a convex set represented by the equations (10.b) below.

Remark 2: For the deterministic discrete-time algebraic Riccati equation (that is, $N=1$ ) with $L=0, Q \geq 0, R=I$, it was shown in [23] that if $\mathcal{R}(X)=0$ (and thus $\left(B^{*} X B+I\right)^{-1}$ exists) then $B^{*} X B+I>0$. The extension of this result for the CARE does not hold as can be seen in the following counterexample. Consider $N=2, A_{1}=1, A_{2}=\frac{1}{2}, B_{1}=B_{2}=1, Q_{1}=Q_{2}=0$, $R_{1}=R_{2}=1, L_{1}=L_{2}=0, p_{11}=\frac{1}{8}, p_{22}=\frac{1}{2}$. Then it can be shown that the CARE has the following four solutions:

$$
\begin{aligned}
& X_{1}^{1}=X_{2}^{1}=0, X_{1}^{2}=1.5562, X_{2}^{2}=-3.42 \\
& X_{1}^{3}=-0.8308, X_{2}^{3}=-0.3999, X_{1}^{4}=-9.4754, X_{2}^{4}=0.3199
\end{aligned}
$$

and it follows that for the second and forth solutions we have

$1+\mathcal{E}_{1}\left(X^{2}\right)=-1.7979,1+\mathcal{E}_{2}\left(X^{2}\right)=0.0681,1+\mathcal{E}_{1}\left(X^{4}\right)=0.0955,1+\mathcal{E}_{2}\left(X^{4}\right)=-3.5778$.

Therefore even with $L_{i}=0, Q_{i} \geq 0, R_{i}=I$, we cannot say that if $X$ is a hermitian solution of the CARE then $I+B_{i}^{*} \mathcal{E}_{i}(X) B_{i}>0$.

We make the following definitions:

Definition 5: We define $\mathcal{S}():. \mathbb{H}^{m, n} \rightarrow \mathbb{H}^{n}, \mathcal{D}():. \mathbb{H}^{n} \rightarrow \mathbb{H}^{m}$ and $\mathcal{F}():. \mathbb{L} \rightarrow \mathbb{H}^{n, m}$ in the following way; for $F=\left(F_{1}, \ldots, F_{N}\right) \in \mathbb{H}^{m, n}$ and $X=\left(X_{1}, \ldots, X_{N}\right)$ in the appropriate domain $\left(\mathbb{H}^{n}\right.$ or $\left.\mathbb{L}\right), \quad \mathcal{S}(F)=\left(\mathcal{S}_{1}(F), \ldots, \mathcal{S}_{N}(F)\right), \quad \mathcal{D}(X)=\left(\mathcal{D}_{1}(X), \ldots, \mathcal{D}_{N}(X)\right) \quad$ and $\mathcal{F}(X)=\left(\mathcal{F}_{1}(X), \ldots, \mathcal{F}_{N}(X)\right)$ are defined, for $i=1, \ldots, N$, as

$$
\begin{aligned}
& \mathcal{S}_{i}(F):=\left(\begin{array}{ll}
I & F_{i}^{*}
\end{array}\right)\left(\begin{array}{cc}
Q_{i} & L_{i} \\
L_{i}^{*} & R_{i}
\end{array}\right)\left(\begin{array}{c}
I \\
F_{i}
\end{array}\right) \\
& \mathcal{D}_{i}(X):=B_{i}^{*} \mathcal{E}_{i}(X) B_{i}+R_{i} \\
& \mathcal{F}_{i}(X):=-\left(B_{i}^{*} \mathcal{E}_{i}(X) B_{i}+R_{i}\right)^{-1}\left(B_{i}^{*} \mathcal{E}_{i}(X) A_{i}+L_{i}^{*}\right) .
\end{aligned}
$$

The proof of the following lemma is straightforward but otherwise long, and therefore will be omitted (see [OZ] or [RV] for similar results).

Lemma 4: Suppose that $X \in \mathbb{L}$ and for some $\widehat{F}=\left(\widehat{F}_{1}, \ldots, \widehat{F}_{N}\right) \in \mathbb{H}^{n, m}$, 
a) $\widehat{X}=\left(\widehat{X}_{1}, \ldots, \widehat{X}_{N}\right) \in \mathbb{H}^{n}$ is hermitian and satisfies for $i=1, \ldots, N$

$$
\widehat{X}_{i}-\left(A_{i}+B_{i} \widehat{F}_{i}\right)^{*} \mathcal{E}_{i}(\widehat{X})\left(A_{i}+B_{i} \widehat{F}_{i}\right)=\mathcal{S}_{i}(\widehat{F}) .
$$

Then, for $i=1, \ldots, N$,

$$
\begin{aligned}
& \left(\widehat{X}_{i}-X_{i}\right)-\left(A_{i}+B_{i} \widehat{F}_{i}\right)^{*} \mathcal{E}_{i}(\widehat{X}-X)\left(A_{i}+B_{i} \widehat{F}_{i}\right)=\mathcal{R}_{i}(X)+ \\
& \left(\widehat{F}_{i}-\mathcal{F}_{i}(X)\right)^{*} \mathcal{D}_{i}(X)\left(\widehat{F}_{i}-\mathcal{F}_{i}(X)\right) .
\end{aligned}
$$

b) Moreover, if $\widehat{X} \in \mathbb{L}$, then for $i=1, \ldots, N$

$$
\begin{aligned}
& \left(\widehat{X}_{i}-X_{i}\right)-\left(A_{i}+B_{i} \mathcal{F}_{i}(\widehat{X})\right)^{*} \mathcal{E}_{i}(\widehat{X}-X)\left(A_{i}+B_{i} \mathcal{F}_{i}(\widehat{X})\right)=\mathcal{R}_{i}(X)+ \\
& \left(\mathcal{F}_{i}(\widehat{X})-\mathcal{F}_{i}(X)\right)^{*} \mathcal{D}_{i}(X)\left(\mathcal{F}_{i}(\widehat{X})-\mathcal{F}_{i}(X)\right)+\left(\widehat{F}_{i}-\mathcal{F}_{i}(\widehat{X})\right)^{*} \mathcal{D}_{i}(\widehat{X})\left(\widehat{F}_{i}-\mathcal{F}_{i}(\widehat{X})\right)
\end{aligned}
$$

c) Furthermore, if $\widehat{Y}=\left(\widehat{Y}_{1}, \ldots, \widehat{Y}_{N}\right) \in \mathbb{H}^{n}$ ishermitian and satisfies, for $i=1, \ldots, N$

$$
\widehat{Y}_{i}-\left(A_{i}+B_{i} \mathcal{F}_{i}(\widehat{X})\right)^{*} \mathcal{E}_{i}(\widehat{Y})\left(A_{i}+B_{i} \mathcal{F}_{i}(\widehat{X})\right)=\mathcal{S}_{i}(\mathcal{F}(\widehat{X}))
$$

then for $i=1, \ldots, N$,

$$
\begin{aligned}
& \left(\widehat{X}_{i}-\widehat{Y}_{i}\right)-\left(A_{i}+B_{i} \mathcal{F}_{i}(\widehat{X})\right)^{*} \mathcal{E}_{i}(\widehat{X}-\widehat{Y})\left(A_{i}+B_{i} \mathcal{F}_{i}(\widehat{X})\right)= \\
& \left(\widehat{F}_{i}-\mathcal{F}_{i}(\widehat{X})\right)^{*} \mathcal{D}_{i}(\widehat{X})\left(\widehat{F}_{i}-\mathcal{F}_{i}(\widehat{X})\right) .
\end{aligned}
$$

We following theorem proves the existence of the maximal solution of (7) in $\mathbb{M}$.

Theorem 1: Suppose that $(A, B)$ is mean square stabilizable and $\mathbb{M} \neq \emptyset$. Then for $l=0,1,2, \ldots$, there exists $X^{l}=\left(X_{1}^{l}, \ldots, X_{N}^{l}\right)$ which satisfies the following properties:

a) $X^{0} \geq X^{1} \geq \cdots \geq X^{l} \geq X$, for arbitrary $X \in \mathbb{M}$;

b) $r_{\sigma}\left(\mathcal{L}^{l}\right)<1$, where $\mathcal{L}^{l}()=.\left(\mathcal{L}_{1}^{l}(),. \ldots, \mathcal{L}_{N}^{l}().\right)$ and for $i=1, \ldots, N$,

$$
\begin{aligned}
\mathcal{L}_{i}^{l}(.) & :=A_{i}^{l *} \mathcal{E}_{i}(.) A_{i}^{l}, \\
A_{i}^{l} & :=A_{i}+B_{i} F_{i}^{l}, \\
F_{i}^{l} & :=\mathcal{F}_{i}\left(X^{l-1}\right) \text { for } l=1,2, \ldots
\end{aligned}
$$

c) $X_{i}^{l}-A_{i}^{l *} \mathcal{E}_{i}\left(X^{l}\right) A_{i}^{l}=\mathcal{S}_{i}\left(F^{l}\right), i=1, \ldots, N$.

Moreover there exists $X^{+}=\left(X_{1}^{+}, \ldots, X_{N}^{+}\right) \in \widehat{\mathbb{M}}$ such that $X^{+} \geq X$ for any $X \in \mathbb{M}$ and $X^{l} \rightarrow X^{+}$as $l \rightarrow \infty$. Furthermore $r_{\sigma}\left(\mathcal{L}^{+}\right) \leq 1$, where $\mathcal{L}^{+}()=.\left(\mathcal{L}_{1}^{+}(),. \ldots, \mathcal{L}_{N}^{+}().\right)$is defined as $\mathcal{L}_{i}^{+}()=.A_{i}^{+*} \mathcal{E}_{i}(.) A_{i}^{+}$, for $i=1, \ldots, N$, and

$$
\begin{aligned}
F_{i}^{+} & =\mathcal{F}_{i}\left(X^{+}\right) \\
A_{i}^{+} & =A_{i}+B_{i} F_{i}^{+} .
\end{aligned}
$$

Proof: Let us apply induction on $l$ to show that equations (9) are satisfied. Consider an arbitrary $X \in \mathbb{M}$. By the hypothesis that $(A, B)$ is mean square stabilizable (see definition 2 ) we can find 
$F^{0}$ such that $r_{\sigma}\left(\mathcal{L}^{0}\right)<1$, where $\mathcal{L}^{0}()=.\left(\mathcal{L}_{1}^{0}(),. \ldots, \mathcal{L}_{N}^{0}().\right)$ and $\mathcal{L}_{i}^{0}()=.A_{i}^{0 *} \mathcal{E}_{i}(.) A_{i}^{0}$ with $A_{i}^{0}=A_{i}+B_{i} F_{i}^{0}$. Thus, from Lemma 3 , there exists a unique $X^{0}=\left(X_{1}^{0}, \ldots, X_{N}^{0}\right) \in \mathbb{H}^{n}$ satisfying

$$
X_{i}^{0}-A_{i}^{0 *} \mathcal{E}_{i}\left(X^{0}\right) A_{i}^{0}=\mathcal{S}_{i}\left(F^{0}\right), i=1, \ldots, N .
$$

Moreover, since $\mathcal{S}\left(F^{0}\right)$ is hermitian, we have that $X^{0}$ is also hermitian. Setting for $i=1, \ldots, N$,

$$
F_{i}:=\mathcal{F}_{i}(X) .
$$

we have from Lemma 4, equation (8.a) that for $i=1, \ldots, N$

$$
\left(X_{i}^{0}-X_{i}\right)-A_{i}^{0 *} \mathcal{E}_{i}\left(X^{0}-X\right) A_{i}^{0}=\mathcal{R}_{i}(X)+\left(F_{i}^{0}-F_{i}\right)^{*} \mathcal{D}_{i}(X)\left(F_{i}^{0}-F_{i}\right)
$$

and since $\mathcal{R}_{i}(X)+\left(F_{i}^{0}-F_{i}\right)^{*} \mathcal{D}_{i}(X)\left(F_{i}^{0}-F_{i}\right) \geq 0, i=1, \ldots, N$ and $r_{\sigma}\left(\mathcal{L}^{0}\right)<1$, we have from Lemma 3 again that $X^{0}-X \geq 0$. This also shows that $X_{i}^{0} \in \mathbb{N}$, since that

$$
\mathcal{D}_{i}\left(X^{0}\right):=B_{i}^{*} \mathcal{E}_{i}\left(X^{0}\right) B_{i}+R_{i} \geq B_{i}^{*} \mathcal{E}_{i}(X) B_{i}+R_{i}=\mathcal{D}_{i}(X)>0,
$$

and thus equations (9) hold for $l=0$. Suppose now that we already have a sequence $\left\{X^{l}\right\}_{l=0}^{k-1}$ satisfying equations (9). Set

$$
\begin{aligned}
F_{i}^{k} & :=\mathcal{F}_{i}\left(X^{k-1}\right) \\
A_{i}^{k} & :=A_{i}+B_{i} F_{i}^{k} .
\end{aligned}
$$

From equation (8.b) in Lemma 4, we get that

$$
\begin{aligned}
& \left(X_{i}^{k-1}-X_{i}\right)-A_{i}^{k *} \mathcal{E}_{i}\left(X^{k-1}-X\right) A_{i}^{k}=\mathcal{R}_{i}(X)+\left(F_{i}^{k}-F_{i}\right)^{*} \mathcal{D}_{i}(X)\left(F_{i}^{k}-F_{i}\right)+ \\
& \left(F_{i}^{k}-F_{i}^{k-1}\right)^{*} \mathcal{D}_{i}\left(X^{k-1}\right)\left(F_{i}^{k}-F_{i}^{k-1}\right) \geq\left(F_{i}^{k}-F_{i}^{k-1}\right)^{*} \mathcal{D}_{i}\left(X^{k-1}\right)\left(F_{i}^{k}-F_{i}^{k-1}\right)
\end{aligned}
$$

and since, by the induction hypothesis, $\mathcal{D}_{i}\left(X^{k-1}\right)>0$ for $i=1, \ldots, N$, we can find $\delta^{k-1}>0$ such that $\mathcal{D}_{i}\left(X^{k-1}\right)>\delta^{k-1} I$. Thus, for $i=1, \ldots, N$,

$$
\left(X_{i}^{k-1}-X_{i}\right)-A_{i}^{k *} \mathcal{E}_{i}\left(X^{k-1}-X\right) A_{i}^{k} \geq \delta^{k-1}\left(F_{i}^{k}-F_{i}^{k-1}\right)^{*}\left(F_{i}^{k}-F_{i}^{k-1}\right) .
$$

and from Lemma $2, r_{\sigma}\left(\mathcal{L}^{k}\right)<1$. Let $X^{k}$ be the unique hermitian solution of (see Lemma 3 and recall that $\mathcal{S}\left(F^{l}\right)$ is hermitian)

$$
X_{i}^{k}-A_{i}^{k *} \mathcal{E}_{i}\left(X^{k}\right) A_{i}^{k}=\mathcal{S}_{i}\left(F^{l}\right), i=1, \ldots, N .
$$

Equation (8.a) in Lemma 4 yields, for $i=1, \ldots, N$,

$$
\left(X_{i}^{k}-X_{i}\right)-A_{i}^{k *} \mathcal{E}_{i}\left(X^{k}-X\right) A_{i}^{k}=\mathcal{R}_{i}(X)+\left(F_{i}^{k}-F_{i}\right)^{*} \mathcal{D}_{i}(X)\left(F_{i}^{k}-F_{i}\right)
$$

and since $r_{\sigma}\left(\mathcal{L}^{k}\right)<1$, we get from Lemma 3 that $X^{k} \geq X$. Thus $\mathcal{D}\left(X^{k}\right) \geq \mathcal{D}(X)>0$, which shows that $X^{k} \in \mathbb{N}$. Equation (8.c) in Lemma 4 yields for $i=1, \ldots, N$

$$
\left(X_{i}^{k-1}-X_{i}^{k}\right)-A_{i}^{k *} \mathcal{E}_{i}\left(X^{k-1}-X^{k}\right) A_{i}^{k}=\left(F_{i}^{k}-F_{i}^{k-1}\right)^{*} \mathcal{D}_{i}\left(X^{k-1}\right)\left(F_{i}^{k}-F_{i}^{k-1}\right),
$$

which shows, from the fact that $r_{\sigma}\left(\mathcal{L}^{k}\right)<1,\left(F_{i}^{k}-F_{i}^{k-1}\right)^{*} \mathcal{D}_{i}\left(X^{k-1}\right)\left(F_{i}^{k}-F_{i}^{k-1}\right)$ is positive 
semi-definite for each $i=1, \ldots, N$, and Lemma 3 , that $X^{k-1} \geq X^{k} \geq X$. This completes the induction argument for equations (9). Since $\left\{X^{l}\right\}_{l=0}^{\infty}$ is a decreasing sequence with $X^{l} \geq X$ for all $l=0,1, \ldots$, we get that thereexists $X^{+}$hermitian such that (see [W1], page 79) $X^{l} \downarrow X^{+}$as $l \rightarrow \infty$. Clearly, $X^{+} \geq X$, and thus $\mathcal{D}\left(X^{+}\right) \geq \mathcal{D}(X)>0$, showing that $X^{+} \in \mathbb{N}$. Moreover, substituting $F_{i}^{l}=\mathcal{F}_{i}\left(X^{l-1}\right)$ in (9.c) and taking the limit as $l \rightarrow \infty$, we get that

$$
0=X_{i}^{+}-\left(A_{i}+\mathcal{F}_{i}\left(X^{+}\right)\right)^{*} \mathcal{E}_{i}\left(X^{+}\right)\left(A_{i}+\mathcal{F}_{i}\left(X^{+}\right)\right)-\mathcal{S}_{i}\left(\mathcal{F}\left(X^{+}\right)\right), i=1, \ldots, N .
$$

Rearranging the terms we obtain for $i=1, \ldots, N$, that

$$
X_{i}^{+}=A_{i}^{*} \mathcal{E}_{i}\left(X^{+}\right) A_{i}+Q_{i}-\left(A_{i}^{*} \mathcal{E}_{i}\left(X^{+}\right) B_{i}+L_{i}\right)\left(B_{i}^{*} \mathcal{E}_{i}\left(X^{+}\right) B_{i}+R_{i}\right)^{-1}\left(B_{i}^{*} \mathcal{E}_{i}\left(X^{+}\right) A_{i}+L_{i}^{*}\right)
$$

that is, $\mathcal{R}\left(X^{+}\right)=0$, showing the desired result. Since $X$ is arbitrary in $\mathbb{M}$, it follows that $X^{+} \geq X$ for all $X \in \mathbb{M}$. Finally notice that since $r_{\sigma}\left(\mathcal{L}^{k}\right)<1$, we get that (see [S], p. 328, for continuity of the eigenvalues on finite dimensional linear operator entries) $r_{\sigma}\left(\mathcal{L}^{+}\right) \leq 1$, where $\mathcal{L}_{i}^{+}()=.A_{i}^{+*} \mathcal{E}_{i}(.) A_{i}^{+}, A_{i}^{+}=A_{i}+B_{i} F_{i}^{+}$and $F_{i}^{+}=\mathcal{F}_{i}\left(X^{+}\right)$.

The next result establishes a link between a LMI (linear matrix inequality) optimization problem and the maximal solution $X^{+}$in $\mathbb{M}$, and can be seen as a generalization of a result presented in [RG], for the case in which $L_{i} \neq 0$ and $Q_{i}$ and $R_{i}$ are indefinite. Suppose that all matrices involved below are real. Consider the following convex programming problem:

$$
\operatorname{maxtr}\left(\sum_{i=1}^{N} X_{i}\right)
$$

subject, for $i=1, \ldots, N$, to

$$
\left[\begin{array}{cc}
-X_{i}+A_{i}^{*} \mathcal{E}_{i}(X) A_{i}+Q_{i} & A_{i}^{*} \mathcal{E}_{i}(X) B_{i}+L_{i} \\
B_{i}^{*} \mathcal{E}_{i}(X) A_{i}+L_{i}^{*} & B_{i}^{*} \mathcal{E}_{i}(X) B_{i}+R_{i}
\end{array}\right] \geq 0, B_{i}^{*} \mathcal{E}_{i}(X) B_{i}+R_{i}>0, X_{i}=X_{i}^{*}
$$

Theorem 2: Suppose that $(A, B)$ is mean square stabilizable. Then there exists $X^{+} \in \widehat{\mathbb{M}}$ such that $X^{+} \geq X$ for all $X \in \mathbb{M}$ if and only if there exists a solution $\widehat{X}$ for the above convex programming problem (10). Moreover, $\widehat{X}=X^{+}$.

Proof: First of all notice that, from Schur complement (see [SSC], page 13), $X=\left(X_{1}, \ldots, X_{N}\right)$ satisfies the restrictions (10.b) if and only if

$$
-X_{i}+A_{i}^{*} \mathcal{E}_{i}(X) A_{i}+Q_{i}-\left(A_{i}^{*} \mathcal{E}_{i}(X) B_{i}+L_{j}\right)\left(B_{i}^{*} \mathcal{E}_{i}(X) B_{i}+R_{i}\right)^{-1}\left(B_{i}^{*} \mathcal{E}_{i}(X) A_{i}+L_{i}^{*}\right) \geq 0
$$

and $B_{i}^{*} \mathcal{E}_{i}(X) B_{i}+R_{i}>0, X_{i}=X_{i}^{*}$ for $i=1, \ldots, N$, that is, if and only if $X \in \mathbb{M}$. Thus if $X^{+} \in \mathbb{M}$ is such that $X^{+} \geq X$ for all $X \in \mathbb{M}$, clearly $\operatorname{tr}\left(X_{1}^{+}+\cdots+X_{N}^{+}\right) \geq \operatorname{tr}$ $\left(X_{1}+\cdots+X_{N}\right)$ for all $X \in \mathbb{M}$ and since $X^{+} \in \widehat{\mathbb{M}} \subset \mathbb{M}$, it follows that $X^{+}$is the solution of the convex programming problem (10). On the other hand, suppose that $\widehat{X}$ is a solution of the convex programming problem (10). Thus $\widehat{X} \in \mathbb{M} \neq \emptyset$ and from Theorem 1 , there exists $X^{+} \in \mathbb{M}$ such that $X^{+} \geq \widehat{X}$. But from the optimality of $\widehat{X}$ and the fact that $\widehat{\mathbb{M}} \subset \mathbb{M}$, 


$$
\operatorname{tr}\left(X_{1}^{+}-\widehat{X}_{1}\right)+\cdots+\operatorname{tr}\left(X_{N}^{+}-\widehat{X}_{N}\right) \leq 0
$$

Since $X_{1}^{+}-\widehat{X}_{1} \geq 0, \ldots, X_{N}^{+}-\widehat{X}_{N} \geq 0$, we have $X_{1}^{+}=\widehat{X}_{1}, \ldots, X_{N}^{+}=\widehat{X}_{N}$.

\section{Stabilizing Solutions}

In this section we shall be interested in finding conditions for the existence of mean square stabilizing solutions for the CARE (7), which will be defined below. Before we do so, we need the following definition:

Definition 6: We say that $X=\left(X_{1}, \ldots, X_{N}\right) \in \mathbb{H}^{n}$ is a mean square stabilizing solution for the CARE (7) if $X \in \mathbb{L}, \mathcal{R}(X)=0$ and $r_{\sigma}(\mathcal{L})<1$, with $\mathcal{L}()=.\left(\mathcal{L}_{1}(),. \ldots, \mathcal{L}_{N}().\right)$ as in (3.b) above and $\Gamma_{i}=A_{i}+B_{i} \mathcal{F}_{i}(X), i=1, \ldots, N$.

The next lemma establishes uniqueness of the mean square stabilizing solution if $\mathbb{M} \neq \emptyset$ (see proof in the Appendix).

Lemma 5: If $\mathbb{M} \neq \emptyset$ then there exists at most one mean square stabilizing solution for the CARE (7), which will coincide with the maximal solution in $\mathbb{M}$.

In what follows, we write

$$
\mathcal{R}(X)^{1 / 2}:=\left(\mathcal{R}_{1}(X)^{1 / 2}, \ldots, \mathcal{R}_{N}(X)^{1 / 2}\right) \text { and } A+B \mathcal{F}(X):=\left(A_{1}+B_{1} \mathcal{F}_{1}(X), \ldots, A_{N}+B_{N} \mathcal{F}_{N}(X)\right) .
$$

Theorem 3: Suppose that $\mathbb{M} \neq \emptyset$. The following assertions are equivalent:

i) $(A, B)$ is mean square stabilizable and $\left(\mathcal{R}(X)^{1 / 2}, A+B \mathcal{F}(X)\right)$ is mean square detectable for some $X \in \mathbb{M}$.

ii) there exists a mean square stabilizing solution to the CARE (7).

Proof: Let us show first that i) implies ii). From the hypothesis that $(A, B)$ is mean square stabilizable and $\mathbb{M} \neq \emptyset$ we get, from Theorem 1, that there exists the maximal solution $X^{+}=\left(X_{1}^{+}, \ldots, X_{N}^{+}\right) \in \widehat{\mathbb{M}}$. Take $X \in \mathbb{M}$ such that $\left(\mathcal{R}(X)^{1 / 2}, A+B \mathcal{F}(X)\right)$ is mean square detectable. Setting $F_{i}^{+}=\mathcal{F}_{i}\left(X^{+}\right), A_{i}^{+}=A_{i}+B_{i} F_{i}^{+}, F_{i}=\mathcal{F}_{i}(X)$, and recalling that

$$
X_{i}^{+}-A_{i}^{+*} \mathcal{E}_{i}(\widehat{X}) A_{i}^{+}=\mathcal{S}_{i}\left(F_{i}^{+}\right), i=1, \ldots, N
$$

we get from Lemma 4, equation (8.a) that for $i=1, \ldots, N$,

$$
\left(X_{i}^{+}-X_{i}\right)-A_{i}^{+*} \mathcal{E}_{i}\left(X^{+}-X\right) A_{i}^{+}=\mathcal{R}_{i}(X)+\left(F_{i}^{+}-F_{i}\right)^{*} \mathcal{D}_{i}(X)\left(F_{i}^{+}-F_{i}\right) .
$$

From the fact that $\mathcal{R}_{i}(X) \geq 0$ and $\mathcal{D}_{i}(X)>0, i=1, \ldots, N$, we get that we can find $\delta>0$ such that for $i=1, \ldots, N$,

$$
\mathcal{R}_{i}(X)+\left(F_{i}^{+}-F_{i}\right)^{*} \mathcal{D}_{i}(X)\left(F_{i}^{+}-F_{i}\right) \geq \delta\left(\mathcal{R}_{i}(X)+\left(F_{i}^{+}-F_{i}\right)^{*}\left(F_{i}^{+}-F_{i}\right)\right) .
$$

From Definition 3 and the mean square detectability hypothesis, we can find $H=\left(H_{1}, \ldots, H_{N}\right) \in \mathbb{H}^{n}$ such that $r_{\sigma}(\mathcal{L})<1$, where $\mathcal{L}()=.\left(\mathcal{L}_{1}(),. \ldots, \mathcal{L}_{N}().\right)$ is as in (3.b) 
above with $\Gamma_{i}=A_{i}+B_{i} F_{i}+H_{i} \mathcal{R}_{i}(X)^{1 / 2}$.Define $\widehat{F}^{+}:=\left(\widehat{F}_{1}^{+}, \ldots, \widehat{F}_{N}^{+}\right) \in \mathbb{H}^{n, n+m}, \widehat{F}:=$ $\left(\widehat{F}_{1}, \ldots, \widehat{F}_{N}\right) \in \mathbb{H}^{n, n+m}, \widehat{B}:=\left(\widehat{B}_{1}, \ldots, \widehat{B}_{N}\right) \in \mathbb{H}^{n+m, n}$ as

$$
\widehat{F}_{i}^{+}:=\left(\begin{array}{c}
0 \\
F_{i}^{+}
\end{array}\right), \widehat{F}_{i}:=\left(\begin{array}{c}
\mathcal{R}_{i}(X)^{1 / 2} \\
F_{i}
\end{array}\right), \widehat{B}_{i}:=\left(\begin{array}{ll}
H_{i} & B_{i}
\end{array}\right) .
$$

Then it is easy to verify that for $i=1, \ldots, N$

$$
\begin{aligned}
& A_{i}+\widehat{B}_{i} \widehat{F}_{i}=A_{i}+B_{i} F_{i}+H_{i} \mathcal{R}_{i}(X)^{1 / 2}=\Gamma_{i} \\
& A_{i}+\widehat{B}_{i} \widehat{F}_{i}^{+}=A_{i}+B_{i} F_{i}^{+}=A_{i}^{+}
\end{aligned}
$$

and from (11) and (12) we get that

$$
\begin{aligned}
& \delta\left(\widehat{F}_{i}^{+}-\widehat{F}_{i}\right)^{*}\left(\widehat{F}_{i}^{+}-\widehat{F}_{i}\right)=\delta\left(\mathcal{R}_{i}(X)+\left(F_{i}^{+}-F_{i}\right)^{*}\left(F_{i}^{+}-F_{i}\right)\right) \leq \\
& \left(X_{i}^{+}-X_{i}\right)-A_{i}^{+*} \mathcal{E}_{i}\left(X^{+}-X\right) A_{i}^{+}=\left(X_{i}^{+}-X_{i}\right)-\left(A_{i}+\widehat{B}_{i} \widehat{F}_{i}^{+}\right)^{*} \mathcal{E}_{i}\left(X^{+}-X\right)\left(A_{i}+\widehat{B}_{i} \widehat{F}_{i}^{+}\right) .
\end{aligned}
$$

Setting $\mathcal{L}^{+}()=.\left(\mathcal{L}_{1}^{+}(),. \ldots, \mathcal{L}_{N}^{+}().\right), \mathcal{L}_{i}^{+}()=.A_{i}^{+^{*}} \mathcal{E}_{i}(.) A_{i}^{+}$, for $i=1, \ldots, N$, and recalling that $X^{+}-X \geq 0$, we get from Lemma 2 that $r_{\sigma}\left(\mathcal{L}^{+}\right)<1$.

Let us show now that ii) implies i). Suppose that $X=\left(X_{1}, \ldots, X_{N}\right) \in \widehat{\mathbb{M}}$ is the mean square stabilizing solution for the CARE (7). Then clearly $(A, B)$ will be mean square stabilizable and $\left(\mathcal{R}(\mathrm{X})^{1 / 2}, A+B \mathcal{F}(X)\right)=(0, A+B \mathcal{F}(X))$ will be mean square detectable.

Next we present some sufficient conditions for existence of the mean square stabilizing solution, when $\mathbb{M} \neq \emptyset$. We will replace in Theorem 3 the mean square detectability condition by conditions based on the observable and non-observable modes of $\left(\mathcal{R}_{i}(X)^{1 / 2}, p_{i i}^{1 / 2}\left(A_{i}+B_{i} \mathcal{F}_{i}(X)\right)\right)$. The results presented here parallel those in $[\mathrm{C}]$. We shall assume from now on that all matrices involved in the CARE (7) are real. We make the following definition.

Definition 7: Consider $\Gamma=\left(\Gamma_{1}, \ldots, \Gamma_{N}\right) \in \mathbb{H}^{n}$, with $r_{\sigma}\left(p_{i i}^{1 / 2} \Gamma_{i}\right)<1, i=1, \ldots, N$. We set $\mathcal{A} \in \mathbb{B}\left(\mathbb{H}^{n}\right)$ in the following way: for $V=\left(V_{1}, \ldots, V_{N}\right), \mathcal{A}(V)=\left(\mathcal{A}_{1}(V), \ldots, \mathcal{A}_{N}(V)\right)$ is defined as,

$$
\mathcal{A}_{i}(V)=\sum_{k=0}^{\infty}\left(p_{i i}^{1 / 2} \Gamma_{i}^{*}\right)^{k}\left(\Gamma_{i}^{*}\left(\sum_{j=1, j \neq i}^{N} p_{i j} V_{j}\right) \Gamma_{i}\right)\left(p_{i i}^{1 / 2} \Gamma_{i}\right)^{k} .
$$

The following result was proved in [C].

Lemma 6: Consider $\mathcal{L}$ and $\mathcal{A}$ as in (3.b) and (13) respectively. The following assertions are equivalent:

i) $r_{\sigma}(\mathcal{L})<1$.

ii) $r_{\sigma}\left(p_{i i}^{1 / 2} \Gamma_{i}\right)<1$, for $i=1, \ldots, N$, and $r_{\sigma}(\mathcal{A})<1$.

iii) $r_{\sigma}\left(p_{i i}^{1 / 2} \Gamma_{i}\right)<1$, for $i=1, \ldots, N$, and $\sum_{k=0}^{\infty} \mathcal{A}^{k}(H)<\infty$ for some $H>0$ in $\mathbb{H}^{n+}$. 
In the next Lemma we use the same notation as in Theorem 3, and set $\mathbb{D}=\{z \in \mathbb{C} ;\|z\|=1\}$ (see proof in the Appendix).

Lemma 7: Suppose that $\mathbb{M} \neq \emptyset,(A, B)$ is mean square stabilizable, and for some $X \in \mathbb{M}$, $\left(\mathcal{R}_{i}(X)^{1 / 2}, p_{i i}^{1 / 2}\left(A_{i}+B_{i} \mathcal{F}_{i}(X)\right)\right)$ has no unobservable modes in $\mathbb{D}$ for $i=1, \ldots, N$. Then $r_{\sigma}\left(p_{i i}^{1 / 2} A_{i}^{+}\right)<1$, for $i=1, \ldots, N$, where $A_{i}^{+}=A_{i}+B_{i} \mathcal{F}_{i}\left(X^{+}\right)$and $X^{+}$is the maximal solution of the CARE (7) in $\mathbb{M}$.

In what follows, we set $p_{i j}^{T}:=P(\theta(T)=j \mid \theta(0)=i)$ and define $\min \{\emptyset\}:=\infty$. Again we use the same notation as in Theorem 3 , and set $\mathbb{E}=\{z \in \mathbb{C} ;\|z\| \leq 1\}$.

Theorem 4: Suppose that $\mathbb{M} \neq \emptyset$ and $(A, B)$ is mean square stabilizable. Suppose also that for some $X \in \mathbb{M}$ and each $i=1, \ldots, N$, one of the conditions below is satisfied:

a) $\left(\mathcal{R}_{i}(X)^{1 / 2}, p_{i i}^{1 / 2}\left(A_{i}+B_{i} \mathcal{F}_{i}(X)\right)\right)$ has no unobservable modesin $\mathbb{E}$, or

b) $\left(\mathcal{R}_{i}(X)^{1 / 2}, p_{i i}^{1 / 2}\left(A_{i}+B_{i} \mathcal{F}_{i}(X)\right)\right)$ has no unobservable modes in $\mathbb{D}, 0$ is not an unobservable mode of $\left(\mathcal{R}_{i}(X)^{1 / 2}, A_{i}+B_{i} \mathcal{F}_{i}(X)\right)$ and

$$
\zeta(i):=\min \left\{T ; p_{i j}^{T}>0 \text { for some } j \in \Upsilon\right\}<\infty
$$

where $\Upsilon:=\{\kappa \in\{1, \ldots, N\} ; \kappa$ satisfies condition $a)\}$.

Then $X^{+}$, the maximal solution of the CARE (7) in $\mathbb{M}$, is the mean square stabilizing solution of the CARE (7). Moreover, $X^{+}-X>0$.

Proof: This proof parallels the one in Theorem 2 of [C]. From the hypothesis made and Theorem 1 , there exists the maximal solution $X^{+} \in \mathbb{M}$, and from Lemma $7, r_{\sigma}\left(p_{i i}^{1 / 2} A_{i}^{+}\right)<1$, for $i=$ $1, \ldots, N$. Set $Z=\left(Z_{1}, \ldots, Z_{N}\right)$ and $H=\left(H_{1}, \ldots, H_{N}\right)$ as:

$$
\begin{aligned}
& Z_{i}^{*}=\left(\begin{array}{ll}
\mathcal{R}_{i}(X)^{1 / 2} & \left(F_{i}^{+}-F_{i}\right)^{*} \mathcal{D}_{i}(X)^{1 / 2}
\end{array}\right) \\
& H_{i}=\sum_{k=0}^{\infty}\left(p_{i i}^{1 / 2} A_{i}^{+*}\right)^{k} Z_{i}^{*} Z_{i}\left(p_{i i}^{1 / 2} A_{i}^{+}\right)^{k} \geq 0
\end{aligned}
$$

Set $\mathcal{A}()=.\left(\mathcal{A}_{1}(),. \ldots, \mathcal{A}_{N}().\right)$ as in (13) above with $\Gamma=A^{+}$. The proof of the steps below can be found in the Appendix (in Step 3 below, $\Upsilon^{c}=\{1, \ldots, N\}-\Upsilon$ ):

Step 1: $\sum_{k=0}^{\infty} \mathcal{A}^{k}(H)<\infty$.

Step 2: if $i \in \Upsilon$ then $H_{i}>0$. 
Step 3: if $i \in \Upsilon^{c}$ then there exist $i_{k} \in \Upsilon^{c}, k=0, \ldots, \zeta(i)-1$, and $j \in \Upsilon$ such that

$$
H_{j}>0 \text { and } \sum_{\ell=0}^{\zeta(i)-k} \mathcal{A}_{i_{k}}^{\ell}(H)>0, k=0, \ldots, \zeta(i)-1 .
$$

Step 4: Set $\zeta=\max \left\{\zeta(i) ; i \in \Upsilon^{c}\right\}$ and $\bar{H}=\sum_{k=0}^{\zeta} \mathcal{A}^{k}(H)$. Then $\bar{H}>0$ and $\sum_{k=0}^{\infty} \mathcal{A}^{k}(\bar{H})<\infty$.

Step 5: $X^{+}-X>0$.

From Lemma 6 and Step 4, $r_{\sigma}\left(\mathcal{L}^{+}\right)<1$. Step 5 completes the proof of the Theorem.

We have the following Corollary, proved in the Appendix.

Corollary 1: Suppose that $\mathbb{M} \neq \emptyset$ and $(A, B)$ is mean square stabilizable. Suppose also that for some $X \in \mathbb{M}$ andeach $i=1, \ldots, N$, one of the conditions below is satisfied:

a) $\left(\mathcal{R}_{i}(X)^{1 / 2}, p_{i i}^{1 / 2}\left(A_{i}+B_{i} \mathcal{F}_{i}(X)\right)\right)$ is observable, or

b) $\left(\mathcal{R}_{i}(X)^{1 / 2}, p_{i i}^{1 / 2}\left(A_{i}+B_{i} \mathcal{F}_{i}(X)\right)\right)$ is detectable, 0 is not an unobservable mode of $\left(\mathcal{R}_{i}(X)^{1 / 2}, A_{i}+B_{i} \mathcal{F}_{i}(X)\right)$, and $\zeta(i)<\infty$.

Then $X^{+}$, the maximal solution of the CARE (7) in $\mathbb{M}$, is the unique solution of the CARE (7) such that $X^{+}-X \geq 0$. Moreover, $X^{+}-X>0$ and $X^{+}$is the mean square stabilizing solution of the CARE (7).

\section{The CARE Associated to the $H_{\infty}$-Control}

In this section we shall consider the coupled algebraic Riccati equations that arise in the $H_{\infty}$ control theory of Markovian jump linear systems (see [CV1]). In this case we do not have that the convex set $\mathbb{M}$ is not empty, so that the results of the previous section cannot be applied directly (see Remark 3 below). However a recursive algorithm for this case can be obtained. This algorithm can be seen as an adaptation of the algorithm presented in [SW] from the deterministic to the Markovian jump case.

Before we present the definition of the $H_{\infty}$-control of Markovian jump linear systems, we need to introduce the following definitions. For an increasing filtration $\left\{\mathfrak{F}_{k}\right\}, k=0,1, \ldots$, defined on a probability space $(\Omega, \mathfrak{F}, \mathcal{P})$, we set $l_{2}^{r}$ as the Hilbert space formed by the sequence of second order random variables $z=(z(0), z(1), \ldots)$ with $z(k) \in \mathbb{C}^{r}$ and $\mathfrak{F}_{k}$ adapted for each $k=0,1, \ldots$, and such that $\|z\|_{2}^{2}:=\sum_{k=0}^{\infty}\|z(k)\|_{2}^{2}<\infty$, where $\|z(k)\|_{2}^{2}:=\mathrm{E}\left(\|z(k)\|^{2}\right)$.This norm comes from the inner product $\langle y ; z\rangle:=\sum_{k=0}^{\infty} \mathrm{E}\left(y(k)^{*} z(k)\right)$, for $y, z \in \mathbb{C}^{r}$. See [CV1] for more details on the construction of the probability $(\Omega, \mathfrak{F}, \mathcal{P})$ and increasing filtration $\left\{\mathfrak{F}_{k}\right\}$. 
Consider the following discrete-time Markovian jump linear system

$$
\begin{aligned}
& x(k+1)=A_{\theta(k)} x(k)+B_{\theta(k)} u(k)+D_{\theta(k)} w(k) \\
& z(k)=M_{\theta(k)} x(k)+J_{\theta(k)} u(k), x(0)=x_{0}, \theta(0)=\theta_{0}
\end{aligned}
$$

with $D=\left(D_{1}, \ldots, D_{N}\right) \in \mathbb{H}^{r, n}, M=\left(M_{1}, \ldots, M_{N}\right) \in \mathbb{H}^{n, s}, J=\left(J_{1}, \ldots, J_{N}\right) \in \mathbb{H}^{m, s}$ and with $J_{i}^{*} J_{i}>0, i=1, \ldots, N$. For simplicity we shall assume that $J_{i}^{*} J_{i}=I$ and that $M_{i}^{*} J_{i}=0$ for $i=1, \ldots, N$. As pointed out in [CV1], it does not represent any loss of generality for the $H_{\infty}$-control problem. For $u=(u(0), u(1), \ldots)$ and $w=(w(0), w(1), \ldots)$, set

$$
X\left(\theta_{0}, x_{0}, u, w\right)=(x(0), x(1), \ldots) \text { and } Z\left(\theta_{0}, x_{0}, u, w\right)=(z(0), z(1), \ldots)
$$

where $x(k)$ and $z(k)$ are given by equations (15). Clearly $X\left(\theta_{0}, ., .,.\right), Z\left(\theta_{0}, ., .,.\right)$ are linear operators.For $F=\left(F_{1}, \ldots, F_{N}\right) \in \mathbb{H}^{n, m}$, consider equations (15) with $u(k)=F_{\theta(k)} x(k)$ and set:

$$
\begin{aligned}
& \overline{\mathcal{L}}(.)=\left(\overline{\mathcal{L}}_{1}(.), \ldots, \overline{\mathcal{L}}_{N}(.)\right), \overline{\mathcal{L}}_{i}(.)=\left(A_{i}+B_{i} F_{i}\right)^{*} \mathcal{E}_{i}(.)\left(A_{i}+B_{i} F_{i}\right) \\
& X_{F}\left(\theta_{0}, w\right)=(0, x(1), \ldots) \\
& Z_{F}\left(\theta_{0}, w\right)=(0, z(1), \ldots)
\end{aligned}
$$

The following lemma was proved in [CV1]:

Lemma 8: $r_{\sigma}(\overline{\mathcal{L}})<1$ if and only if $X_{F}\left(\theta_{0}, w\right) \in l_{2}^{n}$ for any $w \in l_{2}^{r}$.

From this lemma it follows that if $r_{\sigma}(\overline{\mathcal{L}})<1$ then $X_{F}\left(\theta_{0},.\right) \in \mathbb{B}\left(l_{2}^{r} l_{2}^{n}\right)$ and $Z_{F}\left(\theta_{0},.\right) \in \mathbb{B}\left(l_{2}^{r} l_{2}^{s}\right)$. Therefore we can define the $l_{2}$-induced norm of the operator $Z_{F}\left(\theta_{0},.\right)$ in the usual way, that is,

$$
\left\|Z_{F}\left(\theta_{0}, .\right)\right\|=\sup _{w \in l_{2}^{r}} \frac{\left\|Z_{F}\left(\theta_{0}, w\right)\right\|_{2}}{\|w\|_{2}} .
$$

The $H_{\infty}$-control problem of Markovian jump linear systems is defined in the following way:

$\underline{H}_{\underline{\infty}}$-control problem : For a fixed $v>0$, find $F=\left(F_{1}, \ldots, F_{N}\right) \in \mathbb{H}^{n, m}$ which stabilizes $(A, B)$ in the mean square sense and such that $\left\|Z_{F}\left(\theta_{0},.\right)\right\|<v$ for every $\theta_{0}$.

In other words we seek for a feedback controller $u(k)=F_{\theta(k)} x(k)$ which stabilizes the system (15) in the mean square sense and ensures that the $l_{2}$-induced norm from the additive input disturbance to the output is less than the attenuation value $v$. For the case with no jumps $(N=1)$ this definition coincides with the standard $H_{\infty}$-control problem definition.

We have the following Theorem, proved in [CV1], regarding the $H_{\infty}$-control of the discrete-time Markovian jump system given by equation (15). Set $Q_{i}=M_{i}^{*} M_{i}, i=1, \ldots, N$.

Theorem 5: Suppose that $(M, A)$ is mean square detectable and consider $v>0$ fixed. Then there exists $F=\left(F_{1}, \ldots, F_{N}\right) \in \mathbb{H}^{n, m}$ which stabilizes $(A, B)$ in the mean square sense and such that $\left\|Z_{F}\left(\theta_{0},.\right)\right\|<v$ for every $\theta_{0}$ if and only if there exists $P=\left(P_{1}, \ldots, P_{N}\right) \in \mathbb{H}^{n+}$ satisfying the following conditions: 
i) $I-\frac{1}{v^{2}} D_{i}^{*} \mathcal{E}_{i}(P) D_{i}>0, i=1, \ldots, N$.

ii)

$$
\begin{aligned}
& P_{i}=Q_{i}+A_{i}^{*} \mathcal{E}_{i}(P) A_{i}- \\
& A_{i}^{*} \mathcal{E}_{i}(P)\left(\begin{array}{ll}
B_{i} & \frac{1}{v} D_{i}
\end{array}\right)\left[\left(\begin{array}{cc}
I & 0 \\
0 & -I
\end{array}\right)+\left(\begin{array}{c}
B_{i}^{*} \\
\frac{1}{v} D_{i}^{*}
\end{array}\right) \mathcal{E}_{i}(P)\left(\begin{array}{cc}
B_{i} & \frac{1}{v} D_{i}
\end{array}\right)\right]^{-1}\left(\begin{array}{c}
B_{i}^{*} \\
\frac{1}{v} D_{i}^{*}
\end{array}\right) \mathcal{E}_{i}(P) A_{i}= \\
& Q_{i}+\left(A_{i}+B_{i} F_{i}+\frac{1}{v} D_{i} G_{i}\right)^{*} \mathcal{E}_{i}(P)\left(A_{i}+B_{i} F_{i}+\frac{1}{v} D_{i} G_{i}\right)+F_{i}^{*} F_{i}-G_{i}^{*} G_{i}
\end{aligned}
$$

where

$$
\begin{aligned}
F_{i}=- & \left(I+B_{i}^{*} \mathcal{E}_{i}(P) B_{i}+\frac{1}{v^{2}} B_{i}^{*} \mathcal{E}_{i}(P) D_{i}\left(I-\frac{1}{v^{2}} D_{i}^{*} \mathcal{E}_{i}(P) D_{i}\right)^{-1} D_{i}^{*} \mathcal{E}_{i}(P) B_{i}\right)^{-1} \\
& \left(B_{i}^{*}\left(I+\frac{1}{v^{2}} \mathcal{E}_{i}(P) D_{i}\left(I-\frac{1}{v^{2}} D_{i}^{*} \mathcal{E}_{i}(P) D_{i}\right)^{-1} D_{i}^{*}\right) \mathcal{E}_{i}(P) A_{i}\right)= \\
& -\left(I+B_{i}^{*} \mathcal{E}_{i}(P) B_{i}\right)^{-1} B_{i}^{*} \mathcal{E}_{i}(P)\left(A_{i}+\frac{1}{v} D_{i} G_{i}\right) \\
G_{i}= & \left(I-\frac{1}{v^{2}} D_{i}^{*} \mathcal{E}_{i}(P) D_{i}+\frac{1}{v^{2}} D_{i}^{*} \mathcal{E}_{i}(P) B_{i}\left(I+B_{i}^{*} \mathcal{E}_{i}(P) B_{i}\right)^{-1} B_{i}^{*} \mathcal{E}_{i}(P) D_{i}\right)^{-1} \\
& \left(\frac{1}{v} D_{i}^{*}\left(I-\mathcal{E}_{i}(P) B_{i}\left(I+B_{i}^{*} \mathcal{E}_{i}(P) B_{i}\right)^{-1} B_{i}^{*}\right) \mathcal{E}_{i}(P) A_{i}\right)= \\
& \left(I-\frac{1}{v^{2}} D_{i}^{*} \mathcal{E}_{i}(P) D_{i}\right)^{-1}\left(\frac{1}{v} D_{i}^{*}\right) \mathcal{E}_{i}(P)\left(A_{i}+B_{i} F_{i}\right)
\end{aligned}
$$

iii) $r_{\sigma}(\mathcal{L})<1$ where $\mathcal{L}()=.\left(\mathcal{L}_{1}(),. \ldots, \mathcal{L}_{N}().\right)$ is defined as

$$
\mathcal{L}_{i}(.)=\left(A_{i}+B_{i} F_{i}+\frac{1}{v} D_{i} G_{i}\right)^{*} \mathcal{E}_{i}(.)\left(A_{i}+B_{i} F_{i}+\frac{1}{v} D_{i} G_{i}\right)
$$

Remark 3: From equations (16.a) and (16.b) we can see that we cannot apply directly the results of the previous sections.

Remark 4: The condition that $(M, A)$ is mean square detectable (together with mean square stabilizability of $(A, B))$ is only used to guarantee the existence of the mean square stabilizing solution to the CARE (see Theorem 3)

$$
P_{i}=Q_{i}+A_{i}^{*} \mathcal{E}_{i}(P) A_{i}-A_{i}^{*} \mathcal{E}_{i}(P) B_{i}\left(I+B_{i}^{*} \mathcal{E}_{i}(P) B_{i}\right)^{-1} B_{i}^{*} \mathcal{E}_{i}(P) A_{i} .
$$

In view of Theorem $4,(M, A)$ mean square detectability could be replaced by the following condition. For each $i=1, \ldots, N$, one of the following conditions below is satisfied:

a) $\left(Q_{i}^{1 / 2}, p_{i i} A_{i}\right)$ has no unobservable modes in $\mathbb{E}$, or

b) $\left(Q_{i}^{1 / 2}, p_{i i} A_{i}\right)$ has no unobservable modes in $\mathbb{D}, 0$ is not an unobservable mode of $\left(Q_{i}^{1 / 2}, A_{i}\right)$ and $\zeta(i)<\infty$, where $\zeta(i)$ is as in Theorem 4 . 
The next result shows that $F=\left(F_{1}, \ldots, F_{N}\right)$ as defined in (16.c) stabilizes $(A, B)$ in the mean square sense (see proof in the Appendix).

Lemma 9: Suppose that $P=\left(P_{1}, \ldots, P_{N}\right) \in \mathbb{H}^{n+}$ satisfies equations (16) and set $\overline{\mathcal{L}}()=.\left(\overline{\mathcal{L}}_{1}(),. \ldots, \overline{\mathcal{L}_{N}}().\right)$ with $\overline{\mathcal{L}}_{i}()=.\left(A_{i}+B_{i} F_{i}\right)^{*} \mathcal{E}_{i}().\left(A_{i}+B_{i} F_{i}\right)$. Then $r_{\sigma}(\overline{\mathcal{L}})<1$.

For $V=\left(V_{1}, \ldots, V_{N}\right) \in \mathbb{H}^{n+}$ such that $I-\frac{1}{v^{2}} D_{i}^{*} \mathcal{E}_{i}(V) D_{i}>0, i=1, \ldots, N$, set

$$
\begin{aligned}
& \mathcal{M}(V)=\left(\mathcal{M}_{1}(V), \ldots, \mathcal{M}_{N}(V)\right), \mathcal{N}(V)=\left(\mathcal{N}_{1}(V), \ldots, \mathcal{N}_{N}(V)\right) \\
& \mathcal{W}(V)=\left(\mathcal{W}_{1}(V), \ldots, \mathcal{W}_{N}(V), \text { and } \mathcal{V}(V)=\left(\mathcal{V}_{1}(V), \ldots, \mathcal{V}_{N}(V)\right)\right.
\end{aligned}
$$

as

$$
\begin{aligned}
& \mathcal{M}_{i}(V)=Q_{i}+A_{i}^{*} \mathcal{E}_{i}(V) A_{i}- \\
& A_{i}^{*} \mathcal{E}_{i}(V)\left(B_{i} \quad \frac{1}{v} D_{i}\right)\left[\left(\begin{array}{cc}
I & 0 \\
0 & -I
\end{array}\right)+\left(\begin{array}{c}
B_{i}^{*} \\
\frac{1}{v} D_{i}^{*}
\end{array}\right) \mathcal{E}_{i}(V)\left(B_{i} \quad \frac{1}{v} D_{i}\right)\right]^{-1}\left(\begin{array}{c}
B_{i}^{*} \\
\frac{1}{v} D_{i}^{*}
\end{array}\right) \mathcal{E}_{i}(V) A_{i} \\
& \mathcal{W}_{i}(V)=I+B_{i}^{*} \mathcal{E}_{i}(V) B_{i} \\
& \mathcal{N}_{i}(V)=v^{2}\left(I-\frac{1}{v^{2}} D_{i}^{*} \mathcal{E}_{i}(V) D_{i}+\frac{1}{v^{2}} D_{i}^{*} \mathcal{E}_{i}(V) B_{i}\left(I+B_{i}^{*} \mathcal{E}_{i}(V) B_{i}\right)^{-1} B_{i}^{*} \mathcal{E}_{i}(V) D_{i}\right) \\
& \mathcal{V}_{i}(V)=D_{i}^{*}\left(I-\mathcal{E}_{i}(V) B_{i}\left(I+B_{i}^{*} \mathcal{E}_{i}(V) B_{i}\right)^{-1} B_{i}^{*}\right) \mathcal{E}_{i}(V) A_{i} .
\end{aligned}
$$

Straightforward calculations show that for any $i=1, \ldots, N, x_{0} \in \mathbb{C}^{n}, u_{0} \in \mathbb{C}^{m}, w_{0} \in \mathbb{C}^{r}$,

$$
\begin{aligned}
& \left\|M_{i} x_{0}\right\|^{2}+\left\|u_{0}\right\|^{2}-v^{2}\left\|w_{0}\right\|^{2}+\left(A_{i} x_{0}+B_{i} u_{0}+D_{i} w_{0}\right)^{*} \mathcal{E}_{i}(V)\left(A_{i} x_{0}+B_{i} u_{0}+D_{i} w_{0}\right)= \\
& x_{0}^{*} \mathcal{M}_{i}(V) x_{0}+\left\|\mathcal{W}_{i}(V)^{1 / 2}\left(u_{0}+\widehat{u}\left(V, i, x_{0}, w_{0}\right)\right)\right\|^{2}-\left\|\mathcal{N}_{i}(V)^{1 / 2}\left(w_{0}-\widehat{w}\left(V, i, x_{0}\right)\right)\right\|^{2}
\end{aligned}
$$

where,

$$
\begin{aligned}
& \widehat{w}\left(V, i, x_{0}\right)=\mathcal{N}_{i}(V)^{-1} \mathcal{V}_{i}(V) x_{0} \\
& \widehat{u}\left(V, i, x_{0}, w_{0}\right)=\mathcal{W}_{i}(V)^{-1}\left(B_{i}^{*} \mathcal{E}_{i}(V) A_{i} x_{0}+B_{i}^{*} \mathcal{E}_{i}(V) D_{i} w_{0}\right) .
\end{aligned}
$$

The next Theorem is the main result of this section.

Theorem 6: Suppose that either $(M, A)$ is mean square detectable or condition (18) is satisfied. Suppose also that there exists $P=\left(P_{1}, \ldots, P_{N}\right) \in \mathbb{H}^{n+}$ satisfying the conditions (i), (ii), (iii) of Theorem 5 (equations (16)). Set for $\kappa=0,1, \ldots, P^{\kappa+1}=\mathcal{M}\left(P^{k}\right)$, where $P^{0}$ is the maximal solution of equation (17). Then $P^{\kappa}$ converges to $P$ exponentially fast as $\kappa$ goes to infinity.

Proof: First of all motice that, from Lemma 9, $F=\left(F_{1}, \ldots, F_{N}\right)$ as defined in equation (16.c) stabilizes $(A, B)$ in the mean square sense and thus, $(A, B)$ is mean square stabilizable. From mean square detectability or condition (18) we get that there exists the mean square stabilizing solution $P^{0}$ to equation (17), which coincides with the maximal solution. Consider equation (15) with an arbitrary $w=(w(0), \ldots) \in l_{2}^{r}$ and the minimization problem

$$
\min _{u \in \mathbb{U}} \sum_{k=0}^{\infty} \mathrm{E}\left\{\|z(k)\|^{2}\right\}
$$


where $\quad \mathbb{U}:=\left\{u=(u(0), \ldots) \in l_{2}^{m} \quad\right.$ such that $\quad x=(x(0), \ldots) \in l_{2}^{n}$ for every $w=(w(0), \ldots) \in l_{2}^{r}$ where $x$ is given by (15) $\}$. It has been shown in [CV2] (see also [CV1]) that there exists $\mathcal{U}\left(\theta_{0}, . ..\right) \in \mathbb{B}\left(\mathbb{C}^{n} \times l_{2}^{r}, l_{2}^{m}\right)$ such that $\mathcal{U}\left(\theta_{0}, x_{0}, w\right) \in \mathbb{U}$ and $u=(u(0), \ldots)=\mathcal{U}\left(\theta_{0}, x_{0}, w\right)$ solves the minimization problem posed above. For arbitrary $w \in l_{2}^{r}$ and $u \in \mathbb{U}$, we have from equations (15), (19), and (20), that

$$
\begin{aligned}
& \mathrm{E}\left(x_{0}^{*} P_{\theta_{0}} x_{0}\right)-\mathrm{E}\left(x(\nu+1)^{*} P_{\theta(\nu+1)} x(\nu+1)\right)=\sum_{k=0}^{\nu} \mathrm{E}\left(x(k)^{*} P_{\theta(k)} x(k)-\right. \\
& \left.x(k+1)^{*} P_{\theta(k+1)} x(k+1)\right)=\sum_{k=0}^{\nu} \mathrm{E}\left(\mathrm{E}\left(x(k)^{*} P_{\theta(k)} x(k)-x(k+1)^{*} P_{\theta(k+1)} x(k+1) \mid \mathfrak{F}_{k}\right)\right) \\
& =\sum_{k=0}^{\nu} \mathrm{E}\left(x(k)^{*} P_{\theta(k)} x(k)-x(k+1)^{*} \mathcal{E}_{\theta(k)}(P) x(k+1)\right)=\sum_{k=0}^{\nu} \mathrm{E}\left(\|z(k)\|^{2}-v^{2}\|w(k)\|^{2}\right)- \\
& \sum_{k=0}^{\nu} \mathrm{E}\left(\left\|\mathcal{W}_{\theta(k)}(P)^{1 / 2}(u(k)+\widehat{u}(P, \theta(k), x(k), w(k)))\right\|^{2}\right)+ \\
& \sum_{k=0}^{\nu} \mathrm{E}\left(\left\|\mathcal{N}_{\theta(k)}(P)^{1 / 2}(w(k)-\widehat{w}(P, \theta(k), x(k)))\right\|^{2}\right) .
\end{aligned}
$$

Recalling that $u \in \mathbb{U}$ and $w \in l_{2}$ so that $\mathrm{E}\left(\|x(k)\|^{2}\right)$ goes to zero as $k$ goes to infinity, we get that

$$
\begin{gathered}
\sum_{k=0}^{\infty} \mathrm{E}\left(\|z(k)\|^{2}-v^{2}\|w(k)\|^{2}\right)=\sum_{k=0}^{\infty} \mathrm{E}\left(\left\|\mathcal{W}_{\theta(k)}(P)^{1 / 2}(u(k)+\widehat{u}(P, \theta(k), x(k), w(k)))\right\|^{2}\right) \\
+\sum_{k=0}^{\infty} \mathrm{E}\left(\left\|\mathcal{N}_{\theta(k)}(P)^{1 / 2}(w(k)-\widehat{w}(P, \theta(k), x(k)))\right\|^{2}\right)+\mathrm{E}\left(x_{0}^{*} P_{\theta_{0}} x_{0}\right)
\end{gathered}
$$

and it is clear from above that

$$
\begin{gathered}
\mathrm{E}\left(x_{0}^{*} P_{\theta_{0}} x_{0}\right)=\sup _{w \in l_{2}^{r}} \inf _{u \in \mathbb{U}}\left\{\sum_{k=0}^{\infty} \mathrm{E}\left(\|z(k)\|^{2}-v^{2}\|w(k)\|^{2}\right)\right\} \\
=\left\|Z\left(\theta_{0}, x_{0}, \mathcal{U}\left(\theta_{0}, x_{0}, \widehat{w}\right), \widehat{w}\right)\right\|_{2}^{2}-v^{2}\|\widehat{w}\|_{2}^{2}
\end{gathered}
$$

where

$$
\widehat{w}=(\widehat{w}(0), \widehat{w}(1), \ldots), \widehat{w}(k)=\frac{1}{v} G_{\theta(k)} \widehat{x}(k),
$$

$\widehat{u}=\mathcal{U}\left(\theta_{0}, x_{0}, \widehat{w}\right)=(\widehat{u}(0), \widehat{u}(1), \ldots), \widehat{u}(k)=F_{\theta(k)} \widehat{x}(k)$

$\widehat{x}(k+1)=\left(A_{\theta(k)}+B_{\theta(k)} F_{\theta(k)}+\frac{1}{v} D_{\theta(k)} G_{\theta(k)}\right) \widehat{x}(k), \widehat{x}(0)=x_{0}, \theta(0)=\theta_{0}$.

Defining $l_{2}^{r, \kappa}:=\left\{w=(w(0), \ldots) \in l_{2}^{r} ; w(k)=0\right.$ for $\left.k \geq \kappa\right\}$ for $\kappa=0,1, \ldots$, and supposing for the moment that $I-\frac{1}{v^{2}} D_{i}^{*} \mathcal{E}_{i}\left(P^{\kappa-1}\right) D_{i}>0$ for $\kappa>0$, so that $P^{\kappa}=\mathcal{M}\left(P^{k-1}\right)$ is well defined, we get in a similar way as above that

$$
\begin{aligned}
\mathrm{E}\left(x_{0}^{*} P_{\theta_{0}}^{\kappa} x_{0}\right) & =\sup _{w} \quad \inf \left\{l_{2}^{r, \kappa}\left\{\sum_{k=0}^{\infty} \mathrm{E}\left(\|z(k)\|^{2}-v^{2}\|w(k)\|^{2}\right)\right\}\right. \\
& =\left\|Z\left(\theta_{0}, x_{0}, \mathcal{U}\left(\theta_{0}, x_{0}, w^{\kappa}\right), w^{\kappa}\right)\right\|_{2}^{2}-v^{2}\left\|w^{\kappa}\right\|_{2}^{2}
\end{aligned}
$$


where $w^{\kappa}=\left(w^{\kappa}(0), \ldots, w^{\kappa}(\kappa-1), 0, \ldots\right), w^{\kappa}(k)=\mathcal{N}_{\theta(k)}\left(P^{\kappa-k-1}\right)^{-1} \mathcal{V}_{\theta(k)}\left(P^{\kappa-k-1}\right) x(k)$ for $\kappa=1,2, \ldots$, and $u^{\kappa}=\mathcal{U}\left(\theta_{0}, x_{0}, w^{\kappa}\right)=\left(u^{\kappa}(0), u^{\kappa}(1), \ldots\right)$, with

$$
\begin{aligned}
u^{\kappa}(k)=- & \mathcal{W}_{\theta(k)}\left(P^{\kappa-k-1}\right)^{-1} B_{\theta(k)}^{*} \mathcal{E}_{\theta(k)}\left(P^{\kappa-k-1}\right)\left(A_{\theta(k)}+\right. \\
& \left.D_{\theta(k)} \mathcal{N}_{\theta(k)}\left(P^{\kappa-k-1}\right)^{-1} \mathcal{V}_{\theta(k)}\left(P^{\kappa-k-1}\right)\right) x(k) \text { for } k=0, \ldots, \kappa-1 \\
u^{\kappa}(k)=- & \mathcal{W}_{\theta(k)}\left(P^{0}\right)^{-1} B_{\theta(k)}^{*} \mathcal{E}_{\theta(k)}\left(P^{0}\right) A_{\theta(k)} x(k) \text { for } k \geq \kappa .
\end{aligned}
$$

Let us show by induction on $\kappa$ that

$$
P^{\kappa-1} \leq P^{\kappa} \leq P\left(\text { and thus } I-\frac{1}{v^{2}} D_{i}^{*} \mathcal{E}_{i}\left(P^{\kappa}\right) D_{i} \geq I-\frac{1}{v^{2}} D_{i}^{*} \mathcal{E}_{i}(P) D_{i}>0\right) .
$$

For $\kappa=0$ it is clear from (21) and (22) that $P^{0} \leq P$. Suppose now that $P^{k-1} \leq P$, so that $I-\frac{1}{v^{2}} D_{i}^{*} \mathcal{E}_{i}\left(P^{\kappa-1}\right) D_{i}>0$ and $P^{\kappa}=\mathcal{M}\left(P^{k-1}\right)$ is well defined. Since $l_{2}^{r, \kappa-1} \subset l_{2}^{r, \kappa} \subset l_{2}^{r}$ it follows from (21) and (22) again that $P^{\kappa-1} \leq P^{\kappa} \leq P$, showing the induction argument. Set $\widehat{w}^{\kappa}=\left(\widehat{w}^{\kappa}(0), \ldots, \widehat{w}^{\kappa}(\kappa-1), 0, \ldots, 0\right), \widehat{w}^{\kappa}(k)=\widehat{w}(k)$ for $k=0, \ldots, \kappa-1$. From the fact that $r_{\sigma}(\mathcal{L})<1$ (equation (16.e)), we can find $a>0,0<b<1$, such that $\left\|\mathcal{L}^{k}\right\| \leq a b^{k}$. Moreover, we have that (see [CF2], proof of Lemma 1)

$$
\|\widehat{w}(k)\|_{2}^{2}=\mathrm{E}\left(\frac{1}{v}\left\|G_{\theta(k)} \widehat{x}(k)\right\|^{2}\right) \leq c_{1} \mathrm{E}\left(\|\widehat{x}(k)\|^{2}\right) \leq c_{2}\left\|\mathcal{L}^{k}\right\|\left\|x_{0}\right\|_{2}^{2} \leq c_{3} b^{k}\left\|x_{0}\right\|_{2}^{2}
$$

for appropriate constants $c_{1}, c_{2}, c_{3}$. Therefore

$$
\left\|\widehat{w}-\widehat{w}^{\kappa}\right\|_{2}^{2}=\sum_{k=\kappa}^{\infty}\|\widehat{w}(k)\|_{2}^{2} \leq \frac{1}{1-b} c_{3} b^{k}\left\|x_{0}\right\|_{2}^{2} .
$$

Similarly we can show that $\left\|Z\left(\theta_{0}, x_{0}, \mathcal{U}\left(\theta_{0}, x_{0}, \widehat{w}\right), \widehat{w}\right)\right\|_{2} \leq c_{3}^{\prime}\left\|x_{0}\right\|_{2}$ for some appropriate constant $c_{3}^{\prime}>0$. From equation (21) with $x_{0}=0$, we have that

$$
\begin{aligned}
& 0=\sup _{w \in l_{2}^{r}}\left\{\left\|Z\left(\theta_{0}, 0, \mathcal{U}\left(\theta_{0}, 0, w\right), w\right)\right\|_{2}^{2}-v^{2}\|w\|_{2}^{2}\right\} \\
& \quad \geq\left\|Z\left(\theta_{0}, 0, \mathcal{U}\left(\theta_{0}, 0, \widehat{w}-\widehat{w}^{\kappa}\right), \widehat{w}-\widehat{w}^{\kappa}\right)\right\|_{2}^{2}-v^{2}\left\|\widehat{w}-\widehat{w}^{\kappa}\right\|_{2}^{2} .
\end{aligned}
$$

Equations (22), (23) and (24) with $x_{0} \in \mathbb{C}^{n}$ and $\theta_{0}=i$ yield

$$
\begin{aligned}
x_{0}^{*} P_{i}^{\kappa} x_{0} \geq & \left\|Z\left(i, x_{0}, \mathcal{U}\left(i, x_{0}, \widehat{w}^{\kappa}\right), \widehat{w}^{\kappa}\right)\right\|_{2}^{2}-v^{2}\left\|\widehat{w}^{\kappa}\right\|_{2}^{2} \\
\geq & \left\|Z\left(i, x_{0}, \mathcal{U}\left(i, x_{0}, \widehat{w}\right), \widehat{w}\right)-Z\left(i, 0, \mathcal{U}\left(i, 0, \widehat{w}-\widehat{w}^{\kappa}\right), \widehat{w}-\widehat{w}^{\kappa}\right)\right\|_{2}^{2}-v^{2}\|\widehat{w}\|_{2}^{2} . \\
\geq & \left\|Z\left(i, x_{0}, \mathcal{U}\left(i, x_{0}, \widehat{w}\right), \widehat{w}\right)\right\|_{2}^{2}-v^{2}\|\widehat{w}\|_{2}^{2}- \\
& 2\left|\left\langle Z\left(i, x_{0}, \mathcal{U}\left(i, x_{0}, \widehat{w}\right), \widehat{w}\right) ; Z\left(i, 0, \mathcal{U}\left(i, 0, \widehat{w}-\widehat{w}^{\kappa}\right), \widehat{w}-\widehat{w}^{\kappa}\right)\right\rangle\right| \\
\geq & x_{0}^{*} P_{i} x_{0}-2\left\|Z\left(i, x_{0}, \mathcal{U}\left(i, x_{0}, \widehat{w}\right), \widehat{w}\right)\right\|_{2}\left\|Z\left(i, 0, \mathcal{U}\left(i, 0, \widehat{w}-\widehat{w}^{\kappa}\right), \widehat{w}-\widehat{w}^{\kappa}\right)\right\|_{2} \\
\geq & x_{0}^{*} P_{i} x_{0}-c_{4}\left\|x_{0}\right\|_{2}\left\|\widehat{w}-\widehat{w}^{\kappa}\right\|_{2} \geq x_{0}^{*} P_{i} x_{0}-c_{5}\left\|x_{0}\right\|_{2}^{2} b^{\frac{\kappa}{2}}
\end{aligned}
$$

for appropriate positive constants $c_{4}$ and $c_{5}$. Thus 


$$
0 \leq \frac{1}{\left\|x_{0}\right\|_{2}^{2}} x_{0}^{*}\left(P_{i}-P_{i}^{\kappa}\right) x_{0} \leq \varsigma_{5} b^{\frac{\kappa}{2}}
$$

which shows that $P^{\kappa}$ converges to $P$ exponentially fast as $\kappa$ goes to infinity.

In summary, we have the following procedure for deriving a stabilizing solution $P$ satisfying equations (16), whenever it exits:

i) Solve the convex programming problem given by equations (10). Set the solution of this problem as $P^{0}$.

ii) Determine $P^{\kappa}$ through the following iterations: $P^{\kappa+1}=\mathcal{M}\left(P^{\kappa}\right)$.

iii) If $P^{\kappa}$ converges to $P$ as $\kappa$ goes to infinity, then check if $r_{\sigma}(\mathcal{L})<1$, where $\mathcal{L}$ is given by equation (16.e). If it is, then $P$ is the desired solution.

\section{Conclusion}

In this paper we have studied the discrete-time coupled algebraic Riccati equations (CARE) that arise in quadratic optimal control and $H_{\infty}$-control of Markovian jump linear systems. For the equations that arise in the quadratic optimal control problem, we considered the matrix cost to be only hermitian. Contrarily to the deterministic case, the hamiltonian approach, which leads to extended matrix pencils, fails to characterize the solutions of the CARE via the generalized eigenvalues and eigenvectors of the extended matrix pencils. In fact, as illustrated in Remark 2, some results that hold for the usual discrete-time algebraic Riccati equations cannot be extended to the CARE. In this paper we adopted the approach used in [RV] to derive our conditions for the existence of maximal and stabilizing solutions for the CARE. It was shown in Section 3 that under the assumption of a convex set not being empty and mean square stabilizability, there exists the maximal solution for the CARE. This solution can also be characterized as the solution of a convex optimization problem, a result that leads to numerical procedures. In Section 4 necessary and sufficient conditions for the existence of the stabilizing solution were derived, using the concept of mean square detectability. This condition can be replaced by the usual observability and detectability tests for linear systems, leading to sufficient conditions for the existence of the stabilizing solution of the CARE. Section 5 presented a recursive procedure for obtaining a stabilizing solution of the CARE associated to the $H_{\infty}$-control problem, whenever it exits. These results generalize and unify several previous ones presented in the literature of discrete-time coupled Riccati equations of Markovian jump linear systems.

\section{Acknowledgements}

This work was supported in part by $\mathrm{CNPq}$ (Brazilian National Research Council) under the grant 305173/88-0 for the first author and FAPESP (Research Council of the State of São Paulo) under the grants 97/4668-1 for the first and second authors, and 95/4648-5 for the second author.

The authors are also very grateful to the reviewers for the careful and constructive comments which assisted in improving the quality of the paper. 


\section{References}

[BLW] Bittanti, S., Laub, A.J., Willems, J.C., The Riccati Equation, Springer-Verlang, 1991.

[C] Costa, O.L.V, 1996, Mean square stabilizing solutions for discrete-time coupled algebraic Riccati equations IEEE Trans. Automat. Control 41, 593-598.

[CF1] Costa, O.L.V., and Fragoso, M.D., 1993, Stability results for discrete-time linear systems with Markovian jumping parameters, J. Math. Analysis and Applic. 179, 154-178.

[CV1] Costa, O.L.V., and do Val, J.B.R., 1996, Full information $H_{\infty}-$ control for discrete-time infinite Markov jump parameter systems, J. Math. Analysis and Applic., 202, 578-603.

[CF2] Costa, O.L.V., and Fragoso, M.D., 1995, Discrete-time LQ-optimal control problems for infinite Markov jump parameter systems, IEEE Trans. Automat. Control 40, 2076-2088.

[CV2] Costa, O.L.V., and do Val, J.B.R., 1998, Jump LQ-optimal control for discrete-time Markovian systems with stochastic inputs, Stochastic Analysis and Applications 16, 843-858.

[D] Dorato, P., 1993, Theoretical developments in discrete-time control, Automatica 19, 395400 .

[FCS] Fragoso, M.D., Costa, O.L.V., and de Souza, C.E., 1998, A new approach to linearly perturbed Riccati equations arising in stochastic control, J. Applied Mathematics and Optimization 37, 99-126.

[JFL] Ji, Y., Chizeck, H.J., Feng, X., and Loparo, K.A., 1991, Stability and control of discretetime jump linear systems, Control-Theory and Advanced Technology 7, 247-270.

[K] Kubrusly, C.S., 1985, Mean square stability for discrete bounded linear systems in Hilbert space, SIAM J. Control and Optimization 23, 19-29.

[M1] Mariton, M., Jump Linear Systems in Automatic Control, Marcel Dekker, 1990.

[M2] Morozan, T., 1983, Stabilization of some stochastic discrete-time control systems, Stochastic Analysis and Applications 1, 89-116.

[M3] Morozan, T., 1995, Stability and control for linear systems with jump Markov perturbations, Stochastic Analysis and Applications 13, 91-110.

[NS] Naylor, A.W., and Sell, G.R., Linear Operator Theory in Engineering and Science, 2nd ed., Springer-Verlag, 1982.

[OZ] Oostveen, J. and Zwart, H., 1996, Solving the infinite-dimensional discrete-time algebraic Riccati equation using the extended symplectic pencil, Mathematics of Control, Signals and Systems 9, 242-265.

[RG] Rami, M.A., and El Ghaoui, L., 1996, LMI Optimization for nonstandard Riccati equations arising in stochastic control, IEEE Trans. Automat. Control 41, 1666-1671.

[RV] Ran, A.C.M., and Vreugdenhil, R., 1988, Existence and comparison theorems for the algebraic Riccati equations for continuous and discrete-time systems, Linear Algebra and its Applications 99, 63-83. 
[RT] Ran, A.C.M., and Trentelman, H.L., 1993, Linear quadratic problems with indefinite cost for discrete-time systems, SIAM J. Matrix Analysis and Applications 14, 776-797.

[SSC] Saberi, A., Sannuti, P., and Chen, B.M., $H_{2}$-Optimal Control, Prentice Hall, 1995.

[S] Sontag, E.D., Mathematical Control Theory, Springer Verlag, 1990.

[SW] Stoorvogel, A.A., and Weeren, A.J.T.M., 1994, The discrete-time Riccati equation related to the $H_{\infty}$-control problem, IEEE Trans. Automat. Control 39, 686-691.

[W1] Weidmann, J., Linear Operators in Hilbert Spaces, Springer-Verlag, 1980.

[W2] Wimmer, H.K., 1995, On the existence of a least and negative semidefinite solution of the discrete-time algebraic Riccati equation, J. Math. Systems, Estimation, and Control 5, 445-457.

[W3] Wonham, W.M, 1968, On a matrix Riccati equation of stochastic control, SIAM J. Control 6, 681-697.

\section{APPENDIX}

In this appendix we present the proofs of Lemmas 2, 3, 5, 7, 9, Corollary 1, and Steps 1 to 5 in the proof of Theorem 4. For the proof of Lemma 2, we use the following fact:

Remark A1: For any $S=\left(S_{1}, \ldots, S_{N}\right) \in \mathbb{H}^{n}$, we can find $S^{j} \in \mathbb{H}^{n+}, j=1,2,3,4$ such that $S=\left(S^{1}-S^{2}\right)+\sqrt{-1}\left(S^{3}-S^{4}\right)$ (cf. [14]).

Proof of Lemma 2: Set $\overline{\mathcal{T}}=\overline{\mathcal{L}}^{*}$, so that for any $\mathrm{V}=\left(\mathrm{V}_{1}, \ldots, \mathrm{V}_{N}\right) \in \mathbb{H}^{n}$,

$$
\overline{\mathcal{T}}_{j}(V)=\sum_{i=1}^{N} p_{i j} \Lambda_{i} V_{i} \Lambda_{i}^{*}, j=1, \ldots, N .
$$

Note that for arbitrary $\epsilon>0$ and $V=\left(V_{1}, \ldots, V_{N}\right) \geq 0$,

$$
\begin{aligned}
& \left(A_{i}+B_{i} F_{i}\right) V_{i}\left(G_{i}-F_{i}\right)^{*} B_{i}^{*}+B_{i}\left(G_{i}-F_{i}\right) V_{i}\left(A_{i}+B_{i} F_{i}\right)^{*} \\
& \leq \epsilon^{2}\left(A_{i}+B_{i} F_{i}\right) V_{i}\left(A_{i}+B_{i} F_{i}\right)^{*}+\frac{1}{\epsilon^{2}} B_{i}\left(G_{i}-F_{i}\right) V_{i}\left(G_{i}-F_{i}\right)^{*} B_{i}^{*}
\end{aligned}
$$


and combining (A1) with (A2),

$$
\begin{aligned}
0 & \leq \overline{\mathcal{T}}_{j}(V)=\sum_{i=1}^{N} p_{i j} \Lambda_{i} V_{i} \Lambda_{i}^{*} \\
= & \sum_{i=1}^{N} p_{i j}\left(A_{i}+B_{i} F_{i}+B_{i}\left(G_{i}-F_{i}\right)\right) V_{i}\left(A_{i}+B_{i} F_{i}+B_{i}\left(G_{i}-F_{i}\right)\right)^{*} \\
= & \sum_{i=1}^{N} p_{i j}\left[\left(A_{i}+B_{i} F_{i}\right) V_{i}\left(A_{i}+B_{i} F_{i}\right)^{*}+B_{i}\left(G_{i}-F_{i}\right) V_{i}\left(A_{i}+B_{i} F_{i}\right)^{*}+\right. \\
\leq & \left.\left(1+\epsilon^{2}\right) \sum_{i=1}^{N} p_{i j}\left(A_{i}+B_{i} F_{i}\right) V_{i}\left(G_{i}-F_{i}\right)^{*} B_{i}^{*}+B_{i}\left(A_{i}+B_{i}-F_{i}\right) V_{i}\left(G_{i}-F_{i}\right)^{*} B_{i}^{*}\right] \\
= & \left(1+\epsilon^{2}\right) \mathcal{T}_{j}(V)+\left(1+\frac{1}{\epsilon^{2}}\right) \sum_{i=1}^{N} p_{i j} B_{i}\left(G_{i}-F_{i}\right) V_{i}\left(G_{i}-F_{i}\right)^{*} B_{i}^{*}
\end{aligned}
$$

where $\mathcal{Q}(V)=\left(\mathcal{Q}_{1}(V), \ldots, \mathcal{Q}_{N}(V)\right)$ is defined as

$$
\mathcal{Q}_{j}(V)=\sum_{i=1}^{N} p_{i j} B_{i}\left(G_{i}-F_{i}\right) V_{i}\left(G_{i}-F_{i}\right)^{*} B_{i}^{*}
$$

and $\mathcal{T}=\mathcal{L}^{*}$. Since $r_{\sigma}(\mathcal{T})=r_{\sigma}(\mathcal{L})<1$ by hypothesis, we can choose $\epsilon>0$ such that $r_{\sigma}(\tilde{\mathcal{T}})<1$, where $\tilde{\mathcal{T}}()=.\left(1+\epsilon^{2}\right) \mathcal{T}($.$) . Define for t=0,1, \ldots$ the sequences

$$
\begin{array}{cl}
X(t+1)=\overline{\mathcal{T}}(X(t)) & X(0) \in \mathbb{H}^{n+} \\
Y(t+1)=\widetilde{\mathcal{T}}(Y(t))+\widetilde{\mathcal{Q}}(X(t)), & Y(0)=X(0)
\end{array}
$$

with $\widetilde{\mathcal{Q}}()=.\left(1+\frac{1}{\epsilon^{2}}\right) \mathcal{Q}($.$) . Then for t=0,1,2, \ldots$

$$
Y(t) \geq X(t) \geq 0 \text {. }
$$

Indeed, (A5) is immediate from (A4) for $t=0$. Suppose (A5) holds for $t$. Then from (A3)

$$
\begin{aligned}
Y(t+1) & =\widetilde{\mathcal{T}}(Y(t))+\widetilde{\mathcal{Q}}(X(t))=\left(1+\epsilon^{2}\right) \mathcal{T}(Y(t))+\left(1+\frac{1}{\epsilon^{2}}\right) \mathcal{Q}(X(t)) \\
& \geq\left(1+\epsilon^{2}\right) \mathcal{T}(X(t))+\left(1+\frac{1}{\epsilon^{2}}\right) \mathcal{Q}(X(t)) \geq \overline{\mathcal{T}}(X(t))=X(t+1)
\end{aligned}
$$

showing the result for $t+1$. From (A4) it follows that

$$
Y(t)=\widetilde{\mathcal{T}}^{t}(X(0))+\sum_{s=0}^{t-1} \widetilde{\mathcal{T}}^{t-1-s}(\widetilde{\mathcal{Q}}(X(s)))
$$

and taking the1-norm (equation (2.a)) of the above expression, we have that 


$$
\|Y(t)\|_{1} \leq\left\|\widetilde{\mathcal{T}}^{t}\right\|\|X(0)\|_{1}+\sum_{s=0}^{t-1}\left\|\widetilde{\mathcal{T}}^{t-1-s}\right\|\|\widetilde{\mathcal{Q}}(X(s))\|_{1}
$$

Since $r_{\sigma}(\tilde{\mathcal{T}})<1$, it is possible to find $a>0,0<b<1$, such that $\left\|\widetilde{\mathcal{T}}^{s}\right\| \leq a b^{s}$, $s=0,1, \ldots$, andthus,

$$
\|Y(t)\|_{1} \leq a b^{t}\|X(0)\|_{1}+a \sum_{s=0}^{t-1} b^{t-1-s}\|\widetilde{\mathcal{Q}}(X(s))\|_{1} .
$$

Suppose for the moment that $\sum_{s=0}^{\infty}\|\widetilde{\mathcal{Q}}(X(s))\|_{1}<\infty$. Then

$$
\begin{aligned}
\sum_{t=0}^{\infty}\|Y(t)\|_{1} & \leq \frac{a}{1-b}\|X(0)\|_{1}+a \sum_{t=0}^{\infty} \sum_{s=0}^{t-1} b^{t-1-s}\|\widetilde{\mathcal{Q}}(X(s))\|_{1} \\
& =\frac{a}{1-b}\|X(0)\|_{1}+\frac{a}{1-b} \sum_{s=0}^{\infty}\|\widetilde{\mathcal{Q}}(X(s))\|_{1}<\infty
\end{aligned}
$$

and therefore from (A4) and (A5), for any $X(0)=\left(X_{1}(0), \ldots, X_{N}(0)\right) \geq 0$,

$$
0 \leq \sum_{t=0}^{\infty}\left\|\overline{\mathcal{T}}^{t}(X(0))\right\|_{1}=\sum_{t=0}^{\infty}\|X(t)\|_{1} \leq \sum_{t=0}^{\infty}\|Y(t)\|_{1}<\infty .
$$

As pointed out in Remark A1, any $S=\left(S_{1}, \ldots, S_{N}\right) \in \mathbb{H}^{n}$, can be decomposed as

$$
S=\left(S^{1}-S^{2}\right)+\sqrt{-1}\left(S^{3}-S^{4}\right)
$$

for some $S^{i}=\left(S_{1}^{i}, \ldots, S_{N}^{i}\right) \geq 0, i=1,2,3,4$.Therefore,

$$
\sum_{t=0}^{\infty}\left\|\overline{\mathcal{T}}^{t}(S)\right\|_{1} \leq \sum_{i=1}^{4} \sum_{t=0}^{\infty}\left\|\overline{\mathcal{T}}^{t}\left(S^{i}\right)\right\|_{1}<\infty
$$

which shows, from [10] that $r_{\sigma}(\overline{\mathcal{T}})=r_{\sigma}(\overline{\mathcal{L}})<1$. Remains to prove that $\sum_{s=0}^{\infty}$ $\|\widetilde{\mathcal{Q}}(X(s))\|_{1}<\infty$. Indeed, from (5) and setting $c_{0}=\left[\left(1+\frac{1}{\epsilon^{2}}\right) \max _{i}\left\|B_{i}\right\|^{2}\right] \frac{N}{\delta}$, we obtain that 


$$
\begin{aligned}
\|\widetilde{\mathcal{Q}}(X(s))\|_{1} & =\sum_{j=1}^{N}\left\|\widetilde{\mathcal{Q}}_{j}(X(s))\right\| \leq\left(1+\frac{1}{\epsilon^{2}}\right) \sum_{i=1}^{N} \sum_{j=1}^{N} p_{i j}\left\|B_{i}\left(G_{i}-F_{i}\right) X_{i}(s)\left(G_{i}-F_{i}\right)^{*} B_{i}^{k}\right\| \\
& \leq\left(1+\frac{1}{\epsilon^{2}}\right) \sum_{i=1}^{N} \sum_{j=1}^{N} p_{i j}\left\|B_{i}\right\|^{2} \operatorname{tr}\left(\left(G_{i}-F_{i}\right) X_{i}(s)\left(G_{i}-F_{i}\right)^{*}\right) \\
& \leq\left[\left(1+\frac{1}{\epsilon^{2}}\right) \max _{i}\left\|B_{i}\right\|^{2}\right] N \sum_{i=1}^{N} \operatorname{tr}\left(\left\{X_{i}(s)\right\}^{1 / 2}\left(G_{i}-F_{i}\right)^{*}\left(G_{i}-F_{i}\right)\left\{X_{i}(s)\right\}^{1 / 2}\right) \\
& \leq c_{0} \sum_{i=1}^{N} \operatorname{tr}\left(X_{i}(s)\left(P_{i}-\overline{\mathcal{L}}_{i}(P)\right)\right)=c_{0}\langle X(s) ; P-\overline{\mathcal{L}}(P)\rangle \\
& \left.=c_{0}\{\langle X(s) ; P\rangle-\langle X(s) ; \overline{\mathcal{L}}(P)\rangle\}=c_{0}\{\langle X(s) ; P\rangle-\langle\overline{\mathcal{T}}(X(s)) ; P)\rangle\right\} \\
& =c_{0}\{\langle X(s) ; P\rangle-\langle X(s+1) ; P\rangle\} .
\end{aligned}
$$

Taking the sum for $s=0$ to $r$, we get that

$$
\sum_{s=0}^{r}\|\widetilde{\mathcal{Q}}(X(s))\|_{1} \leq c_{0}\{\langle X(0) ; P\rangle-\langle X(r+1) ; P\rangle\} \leq c_{0}\langle X(0) ; P\rangle
$$

since that $P=\left(P_{1}, \ldots, P_{N}\right) \geq 0$ and thus $\langle X(r+1) ; P\rangle \geq 0$. Taking the limit as $r \rightarrow \infty$, we obtain the desired result.

Proof of Lemma 3: Since $r_{\sigma}(\mathcal{L})<1$, we have that (see [22], page 102)

$$
(\mathcal{I}-\mathcal{L})^{-1}(.)=\sum_{j=0}^{\infty} \mathcal{L}^{j}(.)
$$

where $\mathcal{I}$ represents the identity operator. Therefore the unique solution $Y$ of (6) is given by $Y=\sum_{j=0}^{\infty} \mathcal{L}^{j}(S)$ and if $S \in \mathbb{H}^{n *}\left(S \in \mathbb{H}^{n+}, S>0\right.$ respectively $)$ then $Y \in \mathbb{H}^{n *}\left(Y \in \mathbb{H}^{n+}\right.$, $Y>0)$. For the remaining of the proof, see [3].

Proof of Lemma 5: Suppose that $\widehat{X}=\left(\widehat{X}_{1}, \ldots, \widehat{X}_{N}\right)$ is a mean square stabilizing solution for the CARE (7). Clearly $(A, B)$ is mean square stabilizable and since $\mathbb{M} \neq \emptyset$ we get, from Theorem 1, that there exists the maximal solution $X^{+} \in \widehat{\mathbb{M}}$. We have that

$$
\widehat{X}_{i}-\left(A_{i}+B_{i} \mathcal{F}_{i}(\widehat{X})\right)^{*} \mathcal{E}_{i}(\widehat{X})\left(A_{i}+B_{i} \mathcal{F}_{i}(\widehat{X})\right)=\mathcal{S}_{i}(\mathcal{F}(\widehat{X})), i=1, \ldots, N
$$

and from Lemma 4, equation (8.a),

$$
\begin{aligned}
& \left(\widehat{X}_{i}-X_{i}^{+}\right)-\left(A_{i}+B_{i} \mathcal{F}_{i}(\widehat{X})\right)^{*} \mathcal{E}_{i}\left(\widehat{X}-X^{+}\right)\left(A_{i}+B_{i} \mathcal{F}_{i}(\widehat{X})\right)= \\
& \left(\mathcal{F}_{i}(\widehat{X})-\mathcal{F}_{i}\left(X^{+}\right)\right)^{*} \mathcal{D}_{i}\left(X^{+}\right)\left(\mathcal{F}_{i}(\widehat{X})-\mathcal{F}_{i}\left(X^{+}\right)\right), i=1, \ldots, N
\end{aligned}
$$

Since $\mathcal{D}\left(X^{+}\right)>0$ we get that 


$$
\left(\mathcal{F}_{i}(\widehat{X})-\mathcal{F}_{i}\left(X^{+}\right)\right)^{*} \mathcal{D}_{i}\left(X^{+}\right)\left(\mathcal{F}_{i}(\widehat{X})-\mathcal{F}_{i}\left(X^{+}\right)\right) \geq 0, i=1, \ldots, N
$$

and combining the last two equations and recalling that $\widehat{X}$ is a mean square stabilizing solution, we have from Lemma 3 that $\widehat{X}-X^{+} \geq 0$. But this also implies that $\mathcal{D}(\widehat{X}) \geq \mathcal{D}\left(X^{+}\right)>0$ and thus $\widehat{X} \in \widehat{\mathbb{M}}$. From Theorem $1, \widehat{X}-X^{+} \leq 0$, showing the desired result.

Proof of Lemma 7: From the hypothesis made and Theorem 1, it is clear that there exists the maximal solution $X^{+}$for the CARE (7) in $\mathbb{M}$. As seen in Theorem $1, r_{\sigma}\left(\mathcal{L}^{+}\right) \leq 1$ and from this it is easy to verify that $r_{\sigma}\left(p_{i i}^{1 / 2} A_{i}^{+}\right) \leq 1$, for $i=1, \ldots, N$. Indeed, $r_{\sigma}\left(\alpha \mathcal{L}^{+}\right)<1$ for all $\alpha \in(0,1)$ and from Remark $1, r_{\sigma}\left(\left(\alpha p_{i i}\right)^{1 / 2} A_{i}^{+}\right)<1$, for $i=1, \ldots, N$. Taking the limit as $\alpha$ goes up to 1 we have the desired result. Suppose now by contradiction that, for some $i$ and some $\lambda \in \mathbb{C}$, with $\|\lambda\|=1$, and $x \neq 0$ in $\mathbb{C}^{n}, p_{i i}^{1 / 2} A_{i}^{+} x=\lambda x$. From equation (11) we get that

$$
\begin{aligned}
0= & x^{*}\left(\left(X_{i}^{+}-X_{i}\right)-p_{i i} A_{i}^{+*}\left(X_{i}^{+}-X_{i}\right) A_{i}^{+}\right) x=x^{*} A_{i}^{+*}\left(\sum_{j=1, j \neq i}^{N} p_{i j}\left(X_{j}^{+}-X_{j}\right)\right) A_{i}^{+} x+ \\
& x^{*}\left(\mathcal{R}_{i}(X)+\left(F_{i}^{+}-F_{i}\right)^{*} \mathcal{D}_{i}(X)\left(F_{i}^{+}-F_{i}\right)\right) x
\end{aligned}
$$

and since $X^{+}-X \geq 0, \mathcal{D}(X)>0, \mathcal{R}(X) \geq 0$, we can conclude that

$$
\begin{aligned}
& x^{*} A_{i}^{+*}\left(\sum_{j=1, j \neq i}^{N} p_{i j}\left(X_{j}^{+}-X_{j}\right)\right) A_{i}^{+} x=0 \\
& x^{*} \mathcal{R}_{i}(X) x=0, \text { and } x^{*}\left(F_{i}^{+}-F_{i}\right)^{*} \mathcal{D}_{i}(X)\left(F_{i}^{+}-F_{i}\right) x=0
\end{aligned}
$$

which implies that $\mathcal{R}_{i}^{1 / 2}(X) x=0,\left(F_{i}^{+}-F_{i}\right) x=0$. Thus

$$
p_{i i}^{1 / 2}\left(A_{i}+B_{i} F_{i}^{+}\right) x=p_{i i}^{1 / 2}\left(A_{i}+B_{i} F_{i}\right) x=\lambda x \text { and } \mathcal{R}_{i}^{1 / 2}(X) x=0
$$

which is equivalent to saying that $\lambda$ is an unobservable mode of $\left(\mathcal{R}_{i}(\mathrm{X})^{1 / 2}, p_{i i}^{1 / 2}\left(A_{i}+B_{i} \mathcal{F}_{i}(X)\right)\right)$ in $\mathbb{D}$, incontradiction with the hypothesis made.

Proof of step 1 in Theorem 4: We have from equation (11) that

$$
\left(X_{i}^{+}-X_{i}\right)-p_{i i} A_{i}^{+*}\left(X_{i}^{+}-X_{i}\right) A_{i}^{+}=A_{i}^{+*}\left(\sum_{j=1, j \neq i}^{N} p_{i j}\left(X_{j}^{+}-X_{j}\right)\right) A_{i}^{+}+Z_{i}^{*} Z_{i}
$$

and thus

$$
\left(X^{+}-X\right)=H+\mathcal{A}\left(X^{+}-X\right) \geq H \text {. }
$$


Iterating equation (A6) it follows that

$$
X^{+}-X=\mathcal{A}^{t-1}\left(X^{+}-X\right)+\sum_{k=0}^{t} \mathcal{A}^{k}(H) \geq \sum_{k=0}^{t} \mathcal{A}^{k}(H) \geq 0
$$

which shows that $\sum_{k=0}^{\infty} \mathcal{A}^{k}(H)<\infty$.

Proof of step 2 in Theorem 4: From equation (14), it is enough to show that $\left(Z_{i}, p_{i i}^{1 / 2} A_{i}^{+}\right)$is observable. By contradiction, suppose that $\lambda \in \mathbb{C}$ is an unobservable mode of $\left(Z_{i}, p_{i i}^{1 / 2} A_{i}^{+}\right)$, that is, for some $x \neq 0$ in $\mathbb{C}^{n}$,

$$
\begin{aligned}
& p_{i i}^{1 / 2} A_{i}^{+} x=\lambda x \\
& Z_{i} x=\left(\begin{array}{c}
\mathcal{R}_{i}(X)^{1 / 2} x \\
\mathcal{D}_{i}(X)^{1 / 2}\left(F_{i}^{+}-F_{i}\right) x
\end{array}\right)=0
\end{aligned}
$$

which implies that $F_{i} x=F_{i}^{+} x$, and $\mathcal{R}_{i}(X)^{1 / 2} x=0$. Thus we get that

$$
p_{i i}^{1 / 2} A_{i}^{+} x=p_{i i}^{1 / 2}\left(A_{i}+B_{i} F_{i}^{+}\right) x=p_{i i}^{1 / 2}\left(A_{i}+B_{i} F_{i}\right) x=\lambda x, \text { and } \mathcal{R}_{i}(X)^{1 / 2} x=0
$$

that is, $\lambda$ is an unobservable mode of $\left(\mathcal{R}_{i}(\mathrm{X})^{1 / 2}, p_{i i}^{1 / 2}\left(A_{i}+B_{i} F_{i}\right)\right)$. Since $r_{\sigma}\left(p_{i i}^{1 / 2} A_{i}^{+}\right)<1$, we must have that $\|\lambda\|<1$, which is a contradiction with a).

Proof of step 3 in Theorem 4: Suppose now that $i$ satisfies condition b). Set $T=\zeta(i)$ and recall that $\zeta(i)$ represents the minimum time that the Markov chain $\theta(k)$ takes to reach the set $\Upsilon$ starting from the point $i \in \Upsilon^{c}$. Therefore $p_{i j}^{T}>0$ for some $j \in \Upsilon$ and we can find a sequence of distinct elements $\left\{i_{0}, i_{1}, \ldots, i_{T-1}, i_{T}\right\}, i_{0}=i$ and $i_{T}=j$, such that $p_{i i_{1}} p_{i_{1} i_{2}} \ldots p_{i_{T-1} j}>0$ and each $i_{k}, k=0, \ldots, T-1$, satisfies condition b) (otherwise $\zeta(i)$ would not be minimum). Let us show by induction that (recall that $\mathcal{A}^{k}(H)=\left(\mathcal{A}_{1}^{k}(H), \ldots, \mathcal{A}_{N}^{k}(H)\right)$ represents the operator $\mathcal{A}$ applied $k$ times to $H$ )

$$
H_{j}>0, H_{i_{T-1}}+\mathcal{A}_{i_{T-1}}(H)>0, \ldots, H_{i}+\mathcal{A}_{i}(H)+\ldots+\mathcal{A}_{i}^{T}(H)>0 .
$$

As seen in Step 2, $H_{j}>0$. Suppose that $H_{i_{k}}+\mathcal{A}_{i_{k}}(H)+\ldots+\mathcal{A}_{i_{k}}^{T-k}(H)>0$. Let us show that

$$
H_{i_{k-1}}+\mathcal{A}_{i_{k-1}}(H)+\ldots+\mathcal{A}_{i_{k-1}}^{T-k+1}(H)>0 .
$$

Suppose by contradiction that for some $x \neq 0$ in $\mathbb{C}^{n}$,

$$
\left(H_{i_{k-1}}+\mathcal{A}_{i_{k-1}}(H)+\ldots+\mathcal{A}_{i_{k-1}}^{T-k+1}(H)\right) x=0 .
$$

Then we must have that $H_{i_{k-1}} x=0, \ldots, \mathcal{A}_{i_{k-1}}^{T-k+1}(H) x=0$, and thus 


$$
\begin{aligned}
0 & =x^{*}\left(\mathcal{A}_{i_{k-1}}(H)+\ldots+\mathcal{A}_{i_{k-1}}^{T-k+1}(H) x=x^{*} \mathcal{A}_{i_{k-1}}\left(H+\mathcal{A}(H)+\ldots+\mathcal{A}^{T-k}(H) x\right.\right. \\
& =x^{*}\left(\sum_{s=0}^{\infty}\left(p_{i_{k-1} i_{k-1}}^{1 / 2} A_{i_{k-1}}^{+*}\right)^{s} A_{i_{k-1}+*}^{+*}\left(\sum_{l=1, l \neq i_{k-1}}^{N} p_{i_{k-1} l}\left(H_{l}+\ldots+\mathcal{A}_{l}^{T-k}(H)\right)\right) A_{i_{k-1}}^{+}\left(p_{i_{k-1} i_{k-1}}^{1 / 2} A_{i_{k-1}}^{+}\right)^{s}\right) x \\
& \geq x^{*}\left(A_{i_{k-1}}^{+*}\left(\sum_{l=1, l \neq i_{k-1}}^{N} p_{i_{k-1} l}\left(H_{l}+\ldots+\mathcal{A}_{l}^{T-k}(H)\right)\right) A_{i_{k-1}}^{+}\right) x \geq 0
\end{aligned}
$$

and since $p_{i_{k-1} i_{k}}>0, i_{k-1} \neq i_{k}, H_{i_{k}}+\ldots+\mathcal{A}_{i_{k}}^{T-k}(H)>0$, we conclude that $A_{i_{k-1}}^{+} x=0$. Note now that $H_{i_{k-1}} x=0$ implies (see (14)) that $Z_{i_{k-1}} x=0$, and thus $\mathcal{R}_{i_{k-1}}(X) x=0$, and $F_{i_{k-1}}^{+} x=F_{i_{k-1}} x$. Therefore,

$$
A_{i_{k-1}}^{+} x=\left(A_{i_{k-1}}+B_{i_{k-1}} F_{i_{k-1}}^{+}\right) x=\left(A_{i_{k-1}}+B_{i_{k-1}} F_{i_{k-1}}\right) x=0, \text { and } \mathcal{R}_{i_{k-1}}^{1 / 2}(X) x=0
$$

which implies that 0 is an unobservable mode of $\left(\mathcal{R}_{i_{k-1}}(\mathrm{X})^{1 / 2}, A_{i_{k-1}}+B_{i_{k-1}} F_{i_{k-1}}\right)$, contradicting the hypothesis b) of the Theorem.

Proof of steps 4 and 5 in Theorem 4: From Steps 2 and 3,

$$
\bar{H}=\sum_{k=0}^{\zeta} \mathcal{A}^{k}(H)>0,
$$

and thus, from Step 1,

$$
0 \leq \sum_{k=0}^{\infty} \mathcal{A}^{k}(\bar{H})=\sum_{k=0}^{\infty} \mathcal{A}^{k}\left(\sum_{s=0}^{\zeta} \mathcal{A}^{s}(H)\right)=\sum_{k=s}^{\infty} \mathcal{A}^{k}(H) \leq \sum_{k=0}^{\infty} \mathcal{A}^{k}(H)<\infty .
$$

Finally, notice from (A7)and (A8), that $X^{+}-X \geq \sum_{s=0}^{\zeta} \mathcal{A}^{s}(H)>0$.

Proof of Corollary 1: This proof parallels Theorem 3 in [2]. As seen in Theorem $4, X^{+}$is the unique mean square stabilizing solution of the CARE (7) and $X^{+}-X>0$. Suppose that $\bar{X}$ is another solution of the $\operatorname{CARE}$ (7) and that $\bar{X}-X \geq 0$ (thus $\bar{X} \in \widehat{\mathbb{M}}$ ). If we show that $r_{\sigma}\left(A_{i}+B_{i} \mathcal{F}_{i}(\bar{X})\right)<1, i=1, \ldots, N$, then by repeating the same arguments as the proof of Theorem 4 , we get that $\bar{X}$ is the mean square stabilizing solution, and thus $\bar{X}=X^{+}$. Suppose by contradiction that for some $i$ and some $\lambda \in \mathbb{C}$, with $\|\lambda\| \geq 1$, and $x \neq 0$ in $\mathbb{C}^{n}$,

$$
p_{i i}^{1 / 2}\left(A_{i}+B_{i} \mathcal{F}_{i}(\bar{X})\right) x=\lambda x .
$$

Then from Lemma 4, equation (8.a) and setting $\bar{F}_{i}=\mathcal{F}_{i}(\bar{X})$, we get that

$$
\begin{aligned}
& x^{*}\left(\left(\bar{X}_{i}-X_{i}\right)-p_{i i}\left(A_{i}+B_{i} \bar{F}_{i}\right)^{*}\left(\bar{X}_{i}-X_{i}\right)\left(A_{i}+B_{i} \bar{F}_{i}\right)\right) x=\left(1-\|\lambda\|^{2}\right) x^{*}\left(\bar{X}_{i}-X_{i}\right) x= \\
& x^{*}\left(\left(A_{i}+B_{i} \bar{F}_{i}\right)^{*}\left(\sum_{j=1, j \neq i}^{N} p_{i j}\left(\bar{X}_{j}-X_{j}\right)\right)\left(A_{i}+B_{i} \bar{F}_{i}\right)+\mathcal{R}_{i}(X)+\left(\bar{F}_{i}-F_{i}\right)^{*} \mathcal{D}_{i}(X)\left(\bar{F}_{i}-F_{i}\right)\right) x
\end{aligned}
$$

and since $\|\lambda\| \geq 1$ and $\bar{X}-X \geq 0, \mathcal{D}(X)>0, \mathcal{R}(X) \geq 0$, we can conclude that 


$$
\begin{aligned}
& \left(1-\|\lambda\|^{2}\right) x^{*}\left(\bar{X}_{i}-X_{i}\right) x=0 \\
& x^{*}\left(A_{i}+B_{i} \bar{F}_{i}\right)^{*}\left(\sum_{j=1, j \neq i}^{N} p_{i j}\left(\bar{X}_{j}-X_{j}\right)\right)\left(A_{i}+B_{i} \bar{F}_{i}\right) x=0 \\
& x^{*} \mathcal{R}_{i}(X) x=0, \text { and } x^{*}\left(\bar{F}_{i}-F_{i}\right)^{*} \mathcal{D}_{i}(X)\left(\bar{F}_{i}-F_{i}\right) x=0 .
\end{aligned}
$$

Therefore $p_{i i}^{1 / 2}\left(A_{i}+B_{i} \bar{F}_{i}\right) x=p_{i i}^{1 / 2}\left(A_{i}+B_{i} F_{i}\right) x=\lambda x$, and $\mathcal{R}_{i}(X)^{1 / 2} x=0$, which shows that $\lambda$ in an unobservable mode of $\left(\mathcal{R}_{i}(\mathrm{X})^{1 / 2}, p_{i i}^{1 / 2}\left(A_{i}+B_{i} F_{i}\right)\right)$, contradicting a) or b).

Proof of Lemma 9: Setting

$$
\widetilde{F}_{i}=\left(\begin{array}{c}
F_{i} \\
0
\end{array}\right), \widetilde{G}_{i}=\left(\begin{array}{c}
F_{i} \\
G_{i}
\end{array}\right), \widetilde{B}_{i}=\left(\begin{array}{cc}
B_{i} & \frac{1}{v} D_{i}
\end{array}\right)
$$

we get from (16) that for some $\alpha>0$,

$$
P_{i}-\left(A_{i}+\widetilde{B}_{i} \widetilde{F}_{i}\right)^{*} \mathcal{E}_{i}(P)\left(A_{i}+\widetilde{B}_{i} \widetilde{F}_{i}\right) \geq \alpha\left(\widetilde{F}_{i}-\widetilde{G}_{i}\right)^{*}\left(\widetilde{F}_{i}-\widetilde{G}_{i}\right)
$$

and noticing that $\mathcal{L}_{i}()=.\left(A_{i}+\widetilde{B}_{i} \widetilde{G}_{i}\right)^{*} \mathcal{E}_{i}().\left(A_{i}+\widetilde{B}_{i} \widetilde{G}_{i}\right)$ with $r_{\sigma}(\mathcal{L})<1$ (from equation (16.e)), we get from Lemma 2 that $r_{\sigma}(\overline{\mathcal{L}})<1$. 